\title{
THE MULTILINEAR STRONG MAXIMAL FUNCTION
}

\author{
LOUKAS GRAFAKOS, LIGUANG LIU, CARLOS PÉREZ, RODOLFO H. TORRES
}

\begin{abstract}
A multivariable version of the strong maximal function is introduced and a sharp distributional estimate for this operator in the spirit of the Jessen, Marcinkiewicz, and Zygmund theorem is obtained. Conditions that characterize the boundedness of this multivariable operator on products of weighted Lebesgue spaces equipped with multiple weights are obtained. Results for other multi(sub)linear maximal functions associated with bases of open sets are studied too. Bilinear interpolation results between distributional estimates, such as those satisfied by the multivariable strong maximal function, are also proved.
\end{abstract}

\section{INTRODUCTION}

Maximal functions have proved to be tools of great importance in harmonic analysis. Their study not only contains intrinsic interest but also intertwines with the study of singular integral operators, most notably in the context of weighted norm inequalities. Maximal functions have also multi(sub)linear versions that play an equally important role in the study of multilinear operators. Some fundamental linear results are no longer readily available in the multilinear setting, but this shortfall puts in evidence new interesting phenomena that lead to further investigation. Finding appropriate substitutes and alternative tools in the study of multilinear problems is an ongoing project undertaken by a number of researchers. The purpose of this article is to contribute to this endeavor by studying multilinear versions of the strong maximal function and intimately related topics.

Some of the motivation for our work arises from the recent article by Lerner et al [18] where the multisublinear maximal function

$$
\mathcal{M}\left(f_{1}, \ldots, f_{m}\right)(x)=\sup _{\substack{Q \ni x \\ Q \text { cube }}} \prod_{i=1}^{m} \frac{1}{|Q|} \int_{Q}\left|f_{i}\left(y_{i}\right)\right| d y_{i}
$$

Date: March 10, 2011.

1991 Mathematics Subject Classification. Primary 42B20, 42B25. Secondary 46B70, 47B38.

Key words and phrases. Maximal operators, weighted norm inequalities, multilinear singular integrals, Calderón-Zygmund theory, commutators.

The authors would like to acknowledge the support of the following grants. First author: NSF grant DMS 0900946. Third author: Spanish Ministry of Science and Innovation grant MTM200908934. Fourth author: NSF grant DMS 0800492 and a General Research Fund allocation of the University of Kansas. 
associated with cubes with sides parallel to the coordinate axes was introduced. This maximal function is an analogue of the Hardy-Littlewood maximal function and led to the characterization of the class of multiple weights for which multilinear CalderónZygmund operators are bounded on products of weighted Lebesgue spaces. Such operators were introduced by Coifman and Meyer in [2], [3] and were systematically studied in Grafakos and Torres [11] (see also the references therein).

The boundedness

$$
\mathcal{M}: L^{p_{1}}\left(\mathbf{R}^{n}\right) \times \cdots \times L^{p_{m}}\left(\mathbf{R}^{n}\right) \rightarrow L^{p}\left(\mathbf{R}^{n}\right)
$$

whenever

$$
1<p_{1}, \ldots, p_{m} \leq \infty \quad \text { and } \quad \frac{1}{p}=\frac{1}{p_{1}}+\cdots+\frac{1}{p_{m}}
$$

is a simple consequence of Hölder's inequality and of the trivial observation that

$$
\mathcal{M}\left(f_{1}, \ldots, f_{m}\right)(x) \leq \prod_{i=1}^{m} M\left(f_{i}\right),
$$

where $M$ stands for the classical Hardy-Littlewood maximal function. Also, Hölder's inequality for weak spaces yields the appropriate endpoint boundedness, namely

$$
\mathcal{M}: L^{1}\left(\mathbf{R}^{n}\right) \times \cdots \times L^{1}\left(\mathbf{R}^{n}\right) \rightarrow L^{\frac{1}{m}, \infty}\left(\mathbf{R}^{n}\right) .
$$

In this article we study corresponding estimates for the $m$-sublinear version of the strong maximal function, or simply (with a certain abuse of terminology) the strong multilinear maximal function. We define this operator as

$$
\mathcal{M}_{\mathcal{R}}(\vec{f})(x)=\sup _{R \ni x} \prod_{i=1}^{m}\left(\frac{1}{|R|} \int_{R}\left|f_{i}(y)\right| d y\right) \quad x \in \mathbf{R}^{n},
$$

where $\vec{f}=\left(f_{1}, \ldots, f_{m}\right)$ is an $m$-dimensional vector of locally integrable functions and where the supremum is taken over all rectangles with sides parallel to the coordinate axes. Like $\mathcal{M}$, the strong multilinear maximal functions is controlled by the $m$-fold tensor product of the maximal function of each variable. That is,

$$
\mathcal{M}_{\mathcal{R}}(\vec{f}) \leq \prod_{i=1}^{m} M_{\mathcal{R}}\left(f_{i}\right)
$$

where $M_{\mathcal{R}}$ denotes the strong maximal operator on $\mathbf{R}^{n}$ given by

$$
M_{\mathcal{R}}(f)(x)=\sup _{R \ni x} \frac{1}{|R|} \int_{R}|f(y)| d y,
$$

where the supremum is taken over all rectangles with sides parallel to the coordinate axes. Obviously, (1.3) together with Hölder's inequality, yields the appropriate strong type boundedness

$$
\mathcal{M}_{\mathcal{R}}: L^{p_{1}}\left(\mathbf{R}^{n}\right) \times \cdots \times L^{p_{m}}\left(\mathbf{R}^{n}\right) \rightarrow L^{p}\left(\mathbf{R}^{n}\right)
$$


whenever

$$
1<p_{1}, \ldots, p_{m} \leq \infty \quad \text { and } \quad \frac{1}{p}=\frac{1}{p_{1}}+\cdots+\frac{1}{p_{m}} .
$$

However, $M_{\mathcal{R}}$ is not of weak type $(1,1)$; as a substitute, Jessen, Marcinkiewicz and Zygmund [17] showed that there is a constant $C_{n}$ depending only on the dimension $n$ such that for all $f$ on $\mathbf{R}^{n}$,

$$
\left|\left\{x \in \mathbf{R}^{n}: M_{\mathcal{R}}(f)(x)>\lambda\right\}\right| \leq C_{n} \int_{\mathbf{R}^{n}} \Phi_{n}\left(\frac{|f(x)|}{\lambda}\right) d x
$$

where for $t>0$,

$$
\Phi_{n}(t)=t\left(1+\left(\log ^{+} t\right)^{n-1}\right) \approx t(\log (e+t))^{n-1} .
$$

Unlike the case of cubes, in which the classical weak type endpoint of $M$ immediately extends to an estimate for its $m$-fold product on $L^{1} \times \cdots \times L^{1}$ and hence to $\mathcal{M}$, it is not clear how to derive directly an appropriate endpoint estimate for the $m$-fold product of $M_{\mathcal{R}}$ or even $\mathcal{M}_{\mathcal{R}}$. Part of the problem stems from the fact that the Jessen, Marcinkiewicz and Zygmund result is a so-called modular estimate and not a norm estimate.

The geometry of rectangles in $\mathbf{R}^{n}$ is more intricate than that of cubes in $\mathbf{R}^{n}$, even when both classes of sets are restricted to have sides parallel to the axes. The failure of the engulfing property of intersecting rectangles presents a crucial difference between these two classes and signals that the endpoint behavior of the strong maximal function is fundamentally different than that of the Hardy-Littlewood maximal operator. Delicate properties of rectangles in $\mathbf{R}^{n}$ that still make possible the analysis were quantified by Córdoba and Fefferman [5] in their alternative geometric proof of the classical Jessen, Marcinkiewicz, and Zygmund [17] endpoint estimate for the strong maximal function. We investigate the analogous situation in the multilinear setting and look at endpoint distributional estimates. An interesting point is that our result in this direction, Theorem 3.1, says that the operator is more singular as $m$ increases. In addition to the natural interest of the strong maximal function, part of our motivation to study such endpoint results arose also from a question posed in [18] about the analysis of multilinear commutators via multilinear interpolation involving distributional estimates.

Obtaining multilinear estimates from linear ones produces far from optimal results when a theory of weights is considered. Indeed, the pointwise estimate (1.2) is not sharp enough to be used as the starting point for the development of the relevant theory for the maximal function $\mathcal{M}$. The work in [18] put in evidence that there is a much larger class of weights that characterizes the boundedness of $\mathcal{M}$, as well as the boundedness of singular integrals and commutators, than that previously considered by Grafakos and Torres [12] and Pérez and Torres [30]. 
The relevant class of multiple weights for $\mathcal{M}$ is given by the $A_{\vec{P}}$ condition: for $\vec{P}=\left(p_{1}, \cdots, p_{m}\right)$, with $1<p_{1}, \ldots, p_{m}<\infty, \vec{w} \in A_{\vec{P}}$ if

$$
\sup _{Q} \frac{1}{|Q|} \int_{Q} \nu_{\vec{w}}(x) d x \prod_{j=1}^{m}\left(\frac{1}{|Q|} \int_{Q} w_{j}(x)^{1-p_{j}^{\prime}} d x\right)^{\frac{p}{p_{j}^{\prime}}}<\infty
$$

where $\frac{1}{p}=\frac{1}{p_{1}}+\cdots+\frac{1}{p_{m}}$ and

$$
\nu_{\vec{w}}=\prod_{j=1}^{m} w_{j}^{p / p_{j}} .
$$

For $m=1,(1.5)$ goes back to the well-known Muckenhoupt $A_{p}$ condition. It should be remarked that

$$
\prod_{j=1}^{m} A_{p_{j}} \subset A_{\vec{P}}
$$

with strict inclusion; see [18, p.1232 and Remark 7.2]. We address similar questions involving $\mathcal{M}_{\mathcal{R}}$ and more general maximal functions $\mathcal{M}_{\mathcal{B}}$ associated with other bases $\mathcal{B}$. To do so we need to introduce appropriate new classes of multiple weights; we do this in Section 2.

In the linear case, one may often obtain strong weighted estimates from weak type ones using interpolation and the reverse Hölder's property of weights. This approach was adapted by Pérez [23] for general maximal functions. However, a certain complication in the multilinear setting arises and it does not seem to be possible to use interpolation to pass from weak to strong estimates for the aforementioned classes of weights. This complication is bypassed in this article by directly proving strong type estimates using delicate techniques for maximal functions on general bases of open sets adapted from the work of Jawerth and Torchinsky [16] and Jawerth [15].

The main results in this article are the following:

1. A characterization of all $m$-tuples of weights $\vec{w}=\left(w_{1}, \ldots, w_{m}\right)$ for which the multilinear strong maximal function maps

$$
L^{p_{1}}\left(w_{1}\right) \times \cdots \times L^{p_{m}}\left(w_{m}\right) \rightarrow L^{p}\left(\nu_{\vec{w}}\right)
$$

where $\nu_{\vec{w}}=\prod_{j=1}^{m} w_{j}^{p / p_{j}}, 1<p_{1}, \ldots, p_{m}<\infty$, and $\frac{1}{p}=\frac{1}{p_{1}}+\cdots+\frac{1}{p_{m}}$. This characterization requires the notion of the multilinear $A_{\vec{P}}$ condition adapted to rectangles with sides parallel to the axes in $\mathbf{R}^{n}$ and is contained in Theorem 2.5.

2. The $L^{p_{1}}\left(w_{1}\right) \times \cdots \times L^{p_{m}}\left(w_{m}\right) \rightarrow L^{p, \infty}(\nu)$ boundedness of the multilinear strong maximal function, whenever the weights $w_{1}, \ldots, w_{m}$ and an arbitrary $\nu$ satisfy a certain power bump variant of the multilinear $A_{p}$ condition. This is given in Theorem 2.3 , from which a characterization of the weak type inequality in the case $\nu=\nu_{\vec{w}}$ also follows, see Corollary 2.4.

3. A sharp distributional estimate for the multilinear strong maximal operator, analogous to that of Jessen, Marcinkiewicz, and Zygmund. This can be found in Theorem 3.1. We also note that the type of multilinear endpoint distributional estimate 
obtained is very suitable for purposes of interpolation. In fact, we present in Theorem 7.3 a more general version of the bilinear Marcinkiewicz interpolation theorem. We prove strong type bounds for a certain range of exponents starting from weak type bounds and a distributional estimate like the one that the strong maximal function satisfies.

To facilitate the reader's access to each of the independent results contained herein, this article is organized as follows. A discussion of the classical weighted theory and its multilinear extension with respect to general bases is given in Section 2; this also contains the statements of Theorem 2.3 and Theorem 2.5. The proof of the weak boundedness of the strong maximal function in the case of $m+1$ weights (Theorem 2.3) is postponed until Section 4, while the proof of the strong boundedness in the case of $m$ weights (Theorem 2.5) is given in Section 5. The endpoint estimate of Jessen, Marcinkiewicz, and Zygmund and its multilinear extension (Theorem 3.1) are discussed in Section 3. The proof of the latter is presented in Section 6. Finally, the result on bilinear interpolation between distributional estimates (Theorem 7.3) and its application to bilinear commutators are contained in Section 7.

Acknowledgments: The authors would like to thank the referee for valuable remarks.

\section{Classical Weighted theory for a general Basis}

In [21] Muckenhoupt proved a fundamental result characterizing all weights for which the Hardy-Littlewood maximal operator is bounded. As it is nowadays well-known, the surprisingly simple necessary and sufficient condition is the so-called $A_{p}$ condition (cf. (1.5) with $m=1$ ). A different approach to this characterization was found by Jawerth [15] (see Theorem 2.1 below) based on ideas of Sawyer [32]. Recently, a simple and elegant proof of this characterization, which also yields the sharp bound in terms of the $A_{p}$ constant of the weight, was given by Lerner [19].

2.1. The maximal function for a general basis. We start by introducing some notation. By a basis $\mathcal{B}$ in $\mathbf{R}^{n}$ we mean a collection of open sets in $\mathbf{R}^{n}$. We say that $w$ is a weight associated with the basis $\mathcal{B}$ if $w$ is a non-negative measurable function in $\mathbf{R}^{n}$ such that $w(B)=\int_{B} w(y) d y<\infty$ for each $B$ in $\mathcal{B} . M_{\mathcal{B}, w}$ is the corresponding maximal operator defined by

$$
M_{\mathcal{B}, w}(f)(x)=\sup _{\substack{B \ni x \\ B \in \mathcal{B}}} \frac{1}{w(B)} \int_{B}|f(y)| w(y) d y
$$

for $x \in \bigcup_{B \in \mathcal{B}} B$ and $M_{\mathcal{B}, w} f(x)=0$ for $x \notin \bigcup_{B \in \mathcal{B}} B$. If $w \equiv 1$, we simply write $M_{\mathcal{B}} f(x)$.

Several important examples of bases arise by taking $\mathcal{B}=\mathcal{Q}$ the family of all open cubes in $\mathbf{R}^{n}$ with sides parallel to the axes, $\mathcal{B}=\mathcal{D}$ the family of all open dyadic cubes in $\mathbf{R}^{n}$, and $\mathcal{B}=\mathcal{R}$ the family of all open rectangles in $\mathbf{R}^{n}$ with sides parallel to the axes. Other interesting examples are given by bases of open rectangles with sides parallel to the axes and related side lengths. We have for instance the basis $\Re$ formed by all rectangles in $\mathbb{R}^{3}$ with side lengths are $s, t$, and $s t$, for some $t, s>0$. Similarly, 
for a given parameter $N>1$, we consider the the family $\Re_{N}$ of all rectangles in $\mathbb{R}^{2}$ with eccentricity $N$; namely those rectangles whose side lengths are $s$ and $N s$ for some $s>0$. This last basis is associated with the so-called Nikodym maximal function.

A weight $w$ associated with $\mathcal{B}$ is said to satisfy the $A_{p, \mathcal{B}}$ condition, $1<p<\infty$, if

$$
\sup _{B \in \mathcal{B}}\left(\frac{1}{|B|} \int_{B} w d x\right)\left(\frac{1}{|B|} \int_{B} w^{1-p^{\prime}} d x\right)^{\frac{p}{p^{\prime}}}<\infty .
$$

In the limiting case $p=1$ we say that $w$ satisfies the $A_{1, \mathcal{B}}$ if

$$
\left(\frac{1}{|B|} \int_{B} w(y) d y\right) \operatorname{ess.sup}_{B}\left(w^{-1}\right) \leq c
$$

for all $B \in \mathcal{B}$; this is equivalent to saying

$$
M_{\mathcal{B}} w(x) \leq c w(x)
$$

for almost all $x \in \mathbf{R}^{n}$. It follows from these definitions and Hölder's inequality that

$$
A_{p, \mathcal{B}} \subset A_{q, \mathcal{B}}
$$

if $1 \leq p \leq q \leq \infty$. Then it is natural to define the class $A_{\infty, \mathcal{B}}$ by setting

$$
A_{\infty, \mathcal{B}}=\bigcup_{p>1} A_{p, \mathcal{B}}
$$

One reason that this general framework is interesting to consider is the following theorem due to Jawerth [15] (see [19] for a simpler proof).

Theorem 2.1. Let $1<p<\infty$. Suppose that $\mathcal{B}$ is a basis and that $w$ is a weight associated with $\mathcal{B}$, and set $\sigma=w^{1-p^{\prime}}$. Then

$$
\left\{\begin{array}{l}
M_{\mathcal{B}}: L^{p}(w) \rightarrow L^{p}(w) \\
M_{\mathcal{B}}: L^{p^{\prime}}(\sigma) \rightarrow L^{p^{\prime}}(\sigma)
\end{array}\right.
$$

if and only if

$$
\left\{\begin{array}{l}
w \in A_{p, \mathcal{B}} \\
M_{\mathcal{B}, w}: L^{p^{\prime}}(w) \rightarrow L^{p^{\prime}}(w) \\
M_{\mathcal{B}, \sigma}: L^{p}(\sigma) \rightarrow L^{p}(\sigma) .
\end{array}\right.
$$

Theorem 2.1 includes Muckenhoupt's result, mentioned above, that for $1<p<\infty$,

$$
M_{\mathcal{Q}}: L^{p}(d \mu) \rightarrow L^{p}(d \mu)
$$

holds if and only if $d \mu=w(y) d y$, with $w \in A_{p, \mathcal{Q}}$, which is simply the classical $A_{p}$ condition.

A key fact is that the proof of Theorem 2.1 in [15] or [19] completely avoids the (difficult) "reverse Hölder inequality". 
2.2. Muckenhoupt basis. Following [23], we use the following class of bases.

Definition 2.1. We say that $\mathcal{B}$ is a Muckenhoupt basis if for any $1<p<\infty$

$$
M_{\mathcal{B}}: L^{p}(w) \rightarrow L^{p}(w)
$$

for any $w \in A_{p, \mathcal{B}}$.

It is shown in [23] that this definition is equivalent to the following result:

Theorem 2.2. $\mathcal{B}$ is a Muckenhoupt basis if and only if for any $1<p<\infty$,

$$
M_{\mathcal{B}, w}: L^{p}(w) \rightarrow L^{p}(w)
$$

whenever $w \in A_{\infty, \mathcal{B}}$.

Most of the important bases are Muckenhoupt bases, and in particular those mentioned above: $\mathcal{Q}, \mathcal{D}, \mathcal{R}$. The fact that $\mathcal{R}$ is a Muckenhoupt basis can be found in [8]. The basis $\Re$ is also a Muckenhoupt basis as shown by R. Fefferman [7].

2.3. The multisublinear maximal operator for a general basis. We are interested in extending some of the main results in [18] concerning the maximal operator in (1.1) to other bases. We introduce a multisublinear version of the maximal operator $\mathcal{M}_{\mathcal{B}}$ by setting

$$
\mathcal{M}_{\mathcal{B}}\left(f_{1}, \ldots, f_{m}\right)(x)=\sup _{B \ni x} \prod_{i=1}^{m}\left(\frac{1}{|B|} \int_{B}\left|f_{i}(y)\right| d y\right) .
$$

For a basis $\mathcal{B}$ we define the multiple weight $A_{\vec{P}, \mathcal{B}}$ condition as in [18]:

Definition 2.2. Let $1 \leq p_{1}, \ldots, p_{m}<\infty$. Given $\vec{w}=\left(w_{1}, \ldots, w_{m}\right)$, set

$$
\nu_{\vec{w}}=\prod_{i=1}^{m} w_{i}^{p / p_{i}}
$$

We say that the m-tuple of weights $\vec{w}$ satisfies the $A_{\vec{P}, \mathcal{B}}$ condition if

$$
\sup _{B \in \mathcal{B}}\left(\frac{1}{|B|} \int_{B} \nu_{\vec{w}}(x) d x\right) \prod_{j=1}^{m}\left(\frac{1}{|B|} \int_{B} w_{j}(x)^{1-p_{j}^{\prime}} d x\right)^{\frac{p}{p_{j}^{\prime}}}<\infty .
$$

When $p_{j}=1,\left(\frac{1}{|B|} \int_{B} w_{j}^{1-p_{j}^{\prime}}\right)^{1 / p_{j}^{\prime}}$ is understood as $\left(\inf _{B} w_{j}\right)^{-1}$. We use $[\vec{w}]_{A_{\vec{P}, \mathcal{B}}}$ to denote the quantity in (2.4).

2.4. The case of $m+1$ weights: weak type estimates and the power bump condition. In view of the $A_{\vec{P}, \mathcal{B}}$ condition, it is natural to say that the $(m+1)$-tuple of weights $(\nu, \vec{w})$ satisfies the multiple multilinear condition $A_{\vec{P}, \mathcal{B}}$ if

$$
\sup _{B \in \mathcal{B}}\left(\frac{1}{|B|} \int_{B} \nu(x) d x\right) \prod_{j=1}^{m}\left(\frac{1}{|B|} \int_{B} w_{j}(x)^{1-p_{j}^{\prime}} d x\right)^{\frac{p}{p_{j}^{\prime}}}<\infty .
$$


Note that here $\nu$ is not assumed to be the weight $\nu_{\vec{w}}$ determined by $w$ in (2.3). Condition (2.5) is sufficient for the characterization of the weak type estimate in the classical linear case with $\mathcal{B}=\mathcal{Q}$ and also in the multilinear version with the same basis (cf. [18]). It is unknown, however, if it is also sufficient for other bases. For example, it remains an open problem whether this condition suffices for the basis $\mathcal{R}$ even in the linear case.

The following definition gives a stronger condition than (2.5) which is quite useful and is often called power bump condition.

Definition 2.3. We say that the $(m+1)$-tuple of weights $(\nu, \vec{w})$ satisfies a bump $A_{\vec{P}, \mathcal{B}}$ condition if $\nu \in A_{\infty, \mathcal{B}}$ and for some $r>1$,

$$
\sup _{B \in \mathcal{B}} \frac{1}{|B|} \int_{B} \nu(x) d x \prod_{j=1}^{m}\left(\frac{1}{|B|} \int_{B} w_{j}^{\left(1-p_{j}^{\prime}\right) r} d x\right)^{\frac{p}{p_{j}^{\prime} r}}<\infty .
$$

This type of power bump condition appeared for the first time in the work of Neugebauer [22] for $m=1$ and $\mathcal{B}=\mathcal{Q}$, but with an extra power bump in the weight $\nu$. Pérez [25] removed the power from the weight $\nu$ and replaced the power bump in $w$ by a logarithmic bump or a more general type of bump. Such power bump conditions were then used in [26] and [27], and in the work of Cruz-Uribe et al [6] to prove very sharp two-weighted estimates for classical operators. For a general $m$ and the basis $\mathcal{B}=\mathcal{Q}$, Moen $\left[20\right.$, Theorem 2.8] obtained that $\mathcal{M}_{\mathcal{Q}}: L^{p_{1}}\left(w_{1}\right) \times \cdots \times L^{p_{m}}\left(w_{m}\right) \rightarrow L^{p}(\nu)$ provided that $1<p_{1}, \ldots, p_{m}<\infty, \frac{1}{p}=\frac{1}{p_{1}}+\cdots+\frac{1}{p_{m}}$, and $(\nu, \vec{w})$ satisfy the power bump condition (2.6) for some $r>1$.

We have the following result concerning $(m+1)$-tuples of weights.

Theorem 2.3. Let $\mathcal{B}$ be a Muckenhoupt basis and let $\vec{P}=\left(p_{1}, \ldots, p_{m}\right)$ with $1<$ $p_{1}, \ldots, p_{m}<\infty$ and $\frac{1}{p}=\frac{1}{p_{1}}+\cdots+\frac{1}{p_{m}}$. Let $(\nu, \vec{w})$ satisfy the power bump condition (2.6) for some $r>1$, then

$$
\mathcal{M}_{\mathcal{B}}: L^{p_{1}}\left(w_{1}\right) \times \cdots \times L^{p_{m}}\left(w_{m}\right) \rightarrow L^{p, \infty}(\nu) .
$$

When applied to the basis $\mathcal{R}$ and $\nu=\nu_{\vec{w}}$, the above theorem gives the following characterization.

Corollary 2.4. Let $\vec{P}=\left(p_{1}, \ldots, p_{m}\right)$ with $1<p_{1}, \ldots, p_{m}<\infty$ and $\frac{1}{p}=\frac{1}{p_{1}}+\cdots+\frac{1}{p_{m}}$ and let $\vec{w}$ be an $m$-tuple of weights. Then

$$
\mathcal{M}_{\mathcal{R}}: L^{p_{1}}\left(w_{1}\right) \times \cdots \times L^{p_{m}}\left(w_{m}\right) \rightarrow L^{p, \infty}\left(\nu_{\vec{w}}\right) \quad \text { if and only if } \quad \vec{w} \in A_{\vec{P}, \mathcal{R}} .
$$

The proof of the corollary follows known arguments. The necessity of $A_{\vec{P}, \mathcal{R}}$ is quite standard and we omit the details. For the sufficiency we first observe that as in [18, Theorem 3.6], the vector condition (2.4) implies that $\nu_{\vec{w}}$ is in the (linear) $A_{m p, \mathcal{R}}$ class and $w_{i}^{1-p_{i}^{\prime}}$ is in the linear $A_{m p_{i}^{\prime}, \mathcal{R}}$ class. In fact, the arguments used in [18] rely only on the use of Hölder's inequality on the sets were the averages involved in the various $A_{p}$ conditions take place, so the arguments would also apply to any other differentiating basis. Using the reverse Hölder inequality property of the basis of rectangles (see 
the book by García-Cuerva and Rubio de Francia [8] p. 458) we can now "bump" the weights $w_{i}^{1-p_{i}^{\prime}}$ and apply Theorem 2.3.

We observe that the case $p_{1}=\cdots=p_{m}=1$ is excluded in the statements of Theorem 2.3 and Corollary 2.4. The problem for this endpoint case remains open except for the unweighted case, which will be considered in Section 3 below.

Finally, we remark that the proof of the Theorem 2.3 given in Section 4, yields that the $A_{\infty}$ condition assumed on $\nu$ can be replaced by the weaker condition $(\mathrm{A})$ given in Definition 5.2.

\subsection{The case of $m$-weights: strong bounds for the strong maximal function.}

A characterization of the strong type bounds for the strong maximal function is possible in this case and we obtain the following.

Theorem 2.5. Let $1<p_{j}<\infty, j=1, \ldots, m$ and $\frac{1}{p}=\frac{1}{p_{1}}+\cdots+\frac{1}{p_{m}}$. Then

$$
\mathcal{M}_{\mathcal{R}}: L^{p_{1}}\left(w_{1}\right) \times \cdots \times L^{p_{m}}\left(w_{m}\right) \rightarrow L^{p}\left(\nu_{\vec{w}}\right) \quad \text { if and only if } \quad \vec{w} \in A_{\vec{P}, \mathcal{R}} .
$$

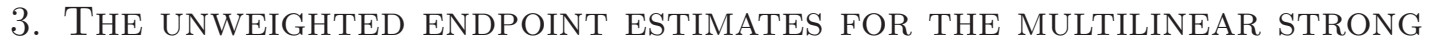 MAXIMAL FUNCTION}

In this section we focus attention on unweighted endpoint properties of the strong multilinear maximal function $\mathcal{M}_{\mathcal{R}}$.

As mentioned in the introduction, (1.3) and Hölder's inequality yield that $\mathcal{M}_{\mathcal{R}}$ is bounded from $L^{p_{1}}\left(\mathbf{R}^{n}\right) \times \cdots \times L^{p_{m}}\left(\mathbf{R}^{n}\right)$ to $L^{p}\left(\mathbf{R}^{n}\right)$ whenever $1<p_{1}, \ldots, p_{m} \leq \infty$ and $\frac{1}{p}=\frac{1}{p_{1}}+\cdots+\frac{1}{p_{m}}$. This argument does not apply to the endpoint case $L^{1} \times \cdots \times L^{1}$ since the operator $M_{\mathcal{R}}$ is not of weak type $(1,1)$.

We have also mentioned a substitute to the weak type $(1,1)$ endpoint estimate for $M_{\mathcal{R}}$ obtained by Jessen, Marcinkiewicz and Zygmund [17], who showed that that for all $f$ on $\mathbf{R}^{n}$

$$
\left|\left\{x \in \mathbf{R}^{n}: M_{\mathcal{R}}(f)(x)>\lambda\right\}\right| \leq C_{n} \int_{\mathbf{R}^{n}} \Phi_{n}\left(\frac{|f(x)|}{\lambda}\right) d x,
$$

where constant $C_{n}$ is a constant depending only on the dimension $n$ and for $t>0$

$$
\Phi_{n}(t)=t\left(1+\left(\log ^{+} t\right)^{n-1}\right) \approx t(\log (e+t))^{n-1} .
$$

Later, Córboda and Fefferman [5] gave a geometric proof of (3.1) answering a question formulated by Zygmund.

These kind of distributional estimates have also appeared in other works in the literature, for instance in [28] for $M^{2}$ (the composition of the maximal function with itself) and for commutators. They are interesting because they provide good endpoint estimates for purposes of interpolation.

As in the linear case, we should not expect weak type estimates when $p_{i}=1$ for all $i$, but rather a distributional estimate involving the function $\Phi_{n}$. Moreover, we will need 
to consider compositions of the function $\Phi_{n}$ with itself. In general, for a given $m \in \mathbb{N}$ and $\Phi$ increasing, we set

$$
\Phi^{(m)}:=\overbrace{\Phi \circ \Phi \circ \cdots \circ \Phi}^{m \text { times }} .
$$

Then $\Phi^{(m)}$ is an increasing function and is also increasing with respect to $m$. In the special case of $\Phi=\Phi_{n}$ we will use the following estimate: there exists a positive constant $C_{n, m}$ which depends on $n$ and $m$ such that for $t>0$,

$$
\Phi_{n}^{(m)}(t) \leq C_{n, m} t[\log (e+t)]^{m(n-1)} .
$$

Theorem 3.1. There exists a positive constant $C$ depending only on $m$ and $n$ such that for all $\lambda>0$,

$$
\left|\left\{x \in \mathbf{R}^{n}: \mathcal{M}_{\mathcal{R}}(\vec{f})(x)>\lambda^{m}\right\}\right| \leq C\left\{\prod_{i=1}^{m} \int_{\mathbf{R}^{n}} \Phi_{n}^{(m)}\left(\frac{\left|f_{i}(x)\right|}{\lambda}\right) d x\right\}^{1 / m}
$$

for all $f_{i}$ on $\mathbf{R}^{n}$ and for all $i=1, \ldots, m$. Furthermore, the theorem is sharp in the sense that we cannot replace $\Phi_{n}^{(m)}$ by $\Phi_{n}^{(k)}$ for $k \leq m-1$.

We present the proof of this result in Section 6 .

4. Proof of the Weak type estimate in the $(m+1)$-Weight CASe

Proof of Theorem 2.3. By homogeneity it is enough to show that

$$
\nu\left(\left\{x \in \mathbf{R}^{n}: \mathcal{M}_{\mathcal{B}}(\vec{f})(x)>1\right\}\right)^{1 / p} \lesssim \prod_{i=1}^{m}\left(\int_{\mathbf{R}^{n}}\left|f_{i}\right|^{p_{i}} w_{i} d x\right)^{1 / p_{i}}
$$

Moreover it is enough to prove (4.1) uniformly for any compact set $K$ contained in the set $\left\{x \in \mathbf{R}^{n}: \mathcal{M}_{\mathcal{B}}(\vec{f})(x)>1\right\}$.

Given such a compact set $K$ we can cover it with a finite collection of sets $\left\{B_{j}\right\}_{j=1}^{N}$ in $\mathcal{B}$ such that

$$
\prod_{i=1}^{m} \frac{1}{\left|B_{j}\right|} \int_{B_{j}}\left|f_{i}\right| d y>1
$$

for all $j$. As in [24] we follow a well-known selection procedure (see for instance [8] p. 463, [4], or [15]). Then we extract a subfamily $\left\{\widetilde{B}_{j}\right\}_{j=1}^{M}$, selected in such a way that $\widetilde{B}_{1}=B_{1}$

$$
\left|\widetilde{B}_{k} \bigcap \bigcup_{j=1}^{k-1} \widetilde{B}_{j}\right|<\left|\widetilde{B}_{k}\right| / 2
$$

and if $B_{l}$ is not in the subfamily $\left\{\widetilde{B}_{j}\right\}_{j=1}^{M}$ then

$$
\left|B_{l} \bigcap \bigcup_{j=1}^{M} \widetilde{B}_{j}\right| \geq\left|B_{l}\right| / 2
$$


Note that if we define

$$
F_{k}=\widetilde{B}_{k} \bigcap \bigcup_{j=1}^{k-1} \widetilde{B}_{j}
$$

then the set

$$
E_{k}=\widetilde{B}_{k} \backslash F_{k}
$$

satisfies

$$
\left|E_{k}\right| \approx\left|\widetilde{B}_{k}\right|
$$

for all $k$ and the sets $\left\{E_{j}\right\}_{j=1}^{M}$ are pairwise disjoint.

We now claim that

$$
\bigcup_{j=1}^{N} B_{j} \subset\left\{x \in \mathbf{R}^{n}: \mathcal{M}_{\mathcal{B}}\left(\vec{\chi}_{\bigcup_{j=1}^{M} \widetilde{B}_{j}}\right)(x) \geq 2^{-m}\right\}
$$

where

$$
\vec{\chi}_{\bigcup_{j=1}^{M} \widetilde{B}_{j}}=\left(\chi_{\bigcup_{j=1}^{M} \widetilde{B}_{j}}, \ldots, \chi_{\bigcup_{j=1}^{M} \widetilde{B}_{j}}\right) .
$$

Assume the claim for a moment and set

$$
\left\{x \in \mathbf{R}^{n}: \mathcal{M}_{\mathcal{B}}\left(\vec{\chi}_{\cup_{j=1}^{M} \widetilde{B}_{j}}\right)(x) \geq 2^{-m}\right\}=G .
$$

Since $\nu \in A_{\infty, \mathcal{B}}, \nu$ is in $A_{m q, \mathcal{B}}$ for some $q>1 / m$. We have

$$
\begin{aligned}
\nu(G) & \leq 2^{m q} \int_{\mathbf{R}^{n}}\left(\prod_{i=1}^{m} M_{\mathcal{B}}\left(\chi_{\bigcup_{j=1}^{M} \widetilde{B}_{j}}\right)\right)^{q} \nu(x) d x \\
& \lesssim \int_{\mathbf{R}^{n}}\left(M_{\mathcal{B}}\left(\chi_{\bigcup_{j=1}^{M} \widetilde{B}_{j}}\right)\right)^{m q} \nu(x) d x \\
& \lesssim \int_{\mathbf{R}^{n}}\left(\chi_{\bigcup_{j=1}^{M} \widetilde{B}_{j}}\right)^{m q} \nu(x) d x \\
& \lesssim \nu\left(\bigcup_{j=1}^{M} \widetilde{B}_{j}\right),
\end{aligned}
$$

and from (4.5) it follows that

$$
\nu(K) \leq \nu\left(\bigcup_{j=1}^{M} B_{j}\right) \lesssim \nu\left(\bigcup_{j=1}^{M} \widetilde{B}_{j}\right) \lesssim \sum_{j=1}^{M} \nu\left(\widetilde{B}_{j}\right)
$$


Now using the bump condition on the weights with some $r>1$, we can estimate the measure of the expression on the right above as follows:

$$
\begin{aligned}
& \sum_{j=1}^{M} \nu\left(\widetilde{B}_{j}\right) \\
\leq & \sum_{j=1}^{M}\left(\prod_{i=1}^{m} \frac{1}{\left|\widetilde{B}_{j}\right|} \int_{\widetilde{B}_{j}}\left|f_{i}\right| d x\right)^{p} \nu\left(\widetilde{B}_{j}\right) \\
\lesssim & \sum_{j=1}^{M} \prod_{i=1}^{m}\left(\frac{1}{\left|\widetilde{B}_{j}\right|} \int_{\widetilde{B}_{j}}\left|f_{i}\right|^{\left(p_{i}^{\prime} r\right)^{\prime}} w_{i}^{\left(p_{i}^{\prime} r\right)^{\prime} / p_{i}} d x\right)^{p /\left(p_{i}^{\prime} r\right)^{\prime}} \prod_{i=1}^{m}\left(\frac{1}{\left|\widetilde{B}_{j}\right|} \int_{\widetilde{B}_{j}} w_{i}^{-p_{i}^{\prime} r / p_{i}} d x\right)^{p / p_{i}^{\prime} r} \nu\left(\widetilde{B}_{j}\right) \\
\lesssim & \sum_{j=1}^{M} \prod_{i=1}^{m}\left(\frac{1}{\left|\widetilde{B}_{j}\right|} \int_{\widetilde{B}_{j}}\left|f_{i}\right|^{\left(p_{i}^{\prime} r\right)^{\prime}} w_{i}^{\left(p_{i}^{\prime} r\right)^{\prime} / p_{i}} d x\right)^{p /\left(p_{i}^{\prime} r\right)^{\prime}}\left|\widetilde{B}_{j}\right|,
\end{aligned}
$$

in view of (2.6). Finally using (4.4), Hölder's inequality with $\sum \frac{p}{p_{i}}=1$, the fact that $p_{i} /\left(p_{i}^{\prime} r\right)^{\prime}>1$, we continue the preceding sequence of inequalities as follows:

$$
\begin{aligned}
& \lesssim \sum_{j=1}^{M} \int_{E_{j}} \prod_{i=1}^{m}\left(M_{\mathcal{B}}\left(\left|f_{i}\right|^{\left(p_{i}^{\prime} r\right)^{\prime}} w_{i}^{\left(p_{i}^{\prime} r\right)^{\prime} / p_{i}}\right)\right)^{p /\left(p_{i}^{\prime} r\right)^{\prime}} d x \\
& \lesssim \int_{\mathbf{R}^{n}} \prod_{i=1}^{m}\left(M_{\mathcal{B}}\left(\left|f_{i}\right|^{\left(p_{i}^{\prime} r\right)^{\prime}} w_{i}^{\left(p_{i}^{\prime} r\right)^{\prime} / p_{i}}\right)\right)^{p /\left(p_{i}^{\prime} r\right)^{\prime}} d x \\
& \lesssim \prod_{i=1}^{m}\left(\int_{\mathbf{R}^{n}}\left(M_{\mathcal{B}}\left(\left|f_{i}\right|^{\left(p_{i}^{\prime} r\right)^{\prime}} w_{i}^{\left(p_{i}^{\prime} r\right)^{\prime} / p_{i}}\right)\right)^{p_{i} /\left(p_{i}^{\prime} r\right)^{\prime}} d x\right)^{p / p_{i}} \\
& \lesssim \prod_{i=1}^{m}\left(\int_{\mathbf{R}^{n}}\left|f_{i}\right|^{p_{i}} w_{i} d x\right)^{p / p_{i}},
\end{aligned}
$$

which gives the desired weak type estimate.

It only remains to verify (4.5). To do so, fix $x$ in $\bigcup_{j} B_{j}$. If $x$ is in one of the sets $\widetilde{B}_{k}$, then $x$ is in $G$ because

$$
\mathcal{M}_{\mathcal{B}}\left(\vec{\chi}_{\bigcup_{j=1}^{M} \widetilde{B}_{j}}\right)(x) \geq \prod_{i=1}^{m} \frac{1}{\left|\widetilde{B}_{k}\right|} \int_{\widetilde{B}_{k}} \chi_{\widetilde{B}_{k}}(y) d y=1 .
$$

On the other hand, if $x \notin \bigcup_{j} \widetilde{B}_{j}$, then $x$ is in some $B_{k}$ satisfying (4.3). It follows then that

$$
\mathcal{M}_{\mathcal{B}}\left(\vec{\chi}_{\bigcup_{j=1}^{M} \widetilde{B}_{j}}\right)(x) \geq \prod_{i=1}^{m} \frac{1}{\left|B_{k}\right|} \int_{B_{k}} \chi_{\bigcup_{j=1}^{M} \widetilde{B}_{j}}(y) d y=\prod_{i=1}^{m} \frac{1}{\left|B_{k}\right|}\left|B_{k} \bigcap \bigcup_{j=1}^{M} \widetilde{B}_{j}\right| \geq 2^{-m},
$$

and so $x$ is also in $G$. The proof of the theorem is now complete. 


\section{The StRong MAXIMAL FUnCtion AND THE $m$-WEIGHT CASE}

The purpose of this section is prove Theorem 2.5 concerning the multilinear strong maximal operator $\mathcal{M}_{\mathcal{R}}$ related to the basis $\mathcal{R}$. To do this we need a special case of a lemma from [15] and some additional definitions for general basis. The first definition concerning the concept of $\alpha$-scattered families is of geometric nature and plays an important role in this context. It has been considered in the works [16] and [15] and implicitly in [4] and [5].

Definition 5.1. Let $\mathcal{B}$ be a basis and let $0<\alpha<1$. A finite sequence $\left\{\tilde{A}_{i}\right\}_{i=1}^{M} \subset \mathcal{B}$ of sets of finite $d x$-measure is called $\alpha$-scattered with respect to the Lebesgue measure if

$$
\left|\tilde{A}_{i} \bigcap \bigcup_{s<i} \tilde{A}_{s}\right| \leq \alpha\left|\tilde{A}_{i}\right|
$$

for all $1<i \leq M$.

Next, we will be considering an important large class of weights.

Definition 5.2. Let $\mathcal{B}$ be a basis and let $w$ be a weight associated to this basis. We say that $w$ satisfies condition $(A)$ if there are constants $0<\lambda<1,0<c(\lambda)<\infty$ such that for all measurable sets $E$ we have

$$
w\left(\left\{x \in \mathbf{R}^{n}: M_{\mathcal{B}}\left(\chi_{E}\right)(x)>\lambda\right\}\right) \leq c(\lambda) w(E) .
$$

This class of weights was also considered in [16] and further exploited in [15]. However, for the Lebesgue measure it goes back to the work in [4] and has been recently considered again by Hagelstein and Stokolos in their articles [13], [14].

One of the reasons that condition $(\mathrm{A})$ is interesting is the fact that it is weaker than the $A_{\infty, \mathcal{B}}$ condition whenever the basis $\mathcal{B}$ is a Muckenhoupt basis. Indeed, if $w \in A_{\infty, \mathcal{B}}$, then $w \in A_{p, \mathcal{B}}$ for some $p>1$ large enough. Then for any measurable set $E$ we have

$$
w\left(\left\{x \in \mathbf{R}^{n}: M_{\mathcal{B}}\left(\chi_{E}\right)(x)>\lambda\right\}\right) \leq \frac{1}{\lambda^{p}} \int M_{\mathcal{B}}\left(\chi_{E}\right)(x)^{p} w(x) d x \leq c(\lambda) w(E),
$$

since $w \in A_{p, \mathcal{B}}$ and the basis $\mathcal{B}$ is a Muckenhoupt basis. It follows that $w$ satisfies condition $(\mathrm{A})$.

Lemma 5.1. Let $\mathcal{B}$ be a basis and let $w$ be a weight associated to this basis. Suppose further that $w$ satisfies condition $(A)$ for some $0<\lambda<1$ and $0<c(\lambda)<\infty$. Then given any finite sequence $\left\{A_{i}\right\}_{i=1}^{M}$ of sets $A_{i} \in \mathcal{B}$,

1) we can find a subsequence $\left\{\tilde{A}_{i}\right\}_{i \in I}$ of $\left\{A_{i}\right\}_{i=1}^{M}$ which is $\lambda$-scattered with respect to the Lebesgue measure;

2) $\tilde{A}_{i}=A_{i}, i \in I$; 
3) for any $1 \leq i<j \leq M+1$

$$
w\left(\bigcup_{s<j} A_{s}\right) \leq c(\lambda)\left[w\left(\bigcup_{s<i} A_{s}\right)+w\left(\bigcup_{i \leq s<j} \tilde{A}_{s}\right)\right],
$$

where $\tilde{A}_{s}=\emptyset$ when $s \notin I$.

The proof of this result is adapted from p. 370 Lemma 1.5 [15].

Proof. We let $\tilde{A}_{1}=A_{1}$ be the first element of the subsequence. Suppose now that $\tilde{A_{1}}, \tilde{A_{i_{2}}}, \cdots, \tilde{A_{i_{l-1}}}$ have been already selected. Then $\tilde{A_{i_{l}}}=A_{i_{l}}$ where $A_{i_{l}}$ is the first element $A$ of $\left\{A_{i}\right\}_{i=1}^{M}$ after $A_{i_{l-1}}$ with the property

$$
\left|A \bigcap \bigcup_{s \leq i_{l-1}} \tilde{A}_{s}\right| \leq \lambda|A|
$$

We continue this way until $\left\{A_{i}\right\}_{i=1}^{M}$ is exhausted. For the subsequence $\left\{\tilde{A}_{i}\right\}_{i \in I}$ obtained in this way we clearly have $\tilde{A}_{i}=A_{i}, i \in I$. We claim that

$$
\bigcup_{s<j} A_{s} \subseteq\left\{x \in \mathbf{R}^{n}: M_{\mathcal{B}}\left(\chi_{E}\right)(x)>\lambda\right\}
$$

where $E=\bigcup_{s<j} \tilde{A}_{s}$. Since $w$ satisfies condition $(A)$ it is easy to deduce immediately (5.1). To prove the claim we first observe that $\bigcup_{s<j} \tilde{A}_{s}$ is trivially contained in the set on the right in (5.2). On the other hand, if $A \in\left\{A_{s}\right\}_{s<j} \backslash\left\{\tilde{A}_{s}\right\}_{s<j}$, then at some index before $j$, A was discarded in the above selection procedure. But this means that $\left|A \cap \bigcup_{s<j_{0}} \tilde{A}_{s}\right|>\lambda|A|$ for some $j_{0} \leq j$ and hence, $A \subseteq\left\{x \in \mathbf{R}^{n}: M_{\mathcal{B}}\left(\chi_{E}\right)(x)>\lambda\right\}$ for $E=\bigcup_{s<j} \tilde{A}_{s}$ verifying the claim. This finishes the proof of the lemma.

Proof of Theorem 2.5. For a vector $\vec{f}$ of bounded functions with compact support consider again the strong multilinear maximal function

$$
\mathcal{M}_{\mathcal{R}}(\vec{f})(x)=\sup _{R \ni x} \prod_{\alpha=1}^{m}\left(\frac{1}{|R|} \int_{R}\left|f_{\alpha}(y)\right| d y\right),
$$

where the supremum is taken over all rectangles $R$ in $\mathbf{R}^{n}$ with sides parallel to the axes. We will use an argument that combines ideas from [18] (second proof of Theorem 3.7), $[15]$ and $[25]$.

Let $N>0$ be a large integer. We will prove the required estimate for the quantity

$$
\int_{2^{-N}<\mathcal{M}_{\mathcal{R}}(\vec{f}) \leq 2^{N+1}} \mathcal{M}_{\mathcal{R}}(\vec{f})(x)^{p} \nu_{\vec{w}}(x) d x
$$

with a bound independent of $N$. For each integer $k,|k| \leq N$, we find a compact set

$$
K_{k} \subset\left\{\mathcal{M}_{\mathcal{R}}(\vec{f})>2^{k}\right\}
$$


satisfying

$$
\nu_{\vec{w}}\left(K_{k}\right) \leq \nu_{\vec{w}}\left(\left\{\mathcal{M}_{\mathcal{R}}(\vec{f})>2^{k}\right\}\right) \leq 2 \nu_{\vec{w}}\left(K_{k}\right)
$$

and a finite sequence $b_{k}=\left\{B_{j}^{k}\right\}_{j \geq 1}$ of sets $B_{j}^{k} \in \mathcal{R}$ with

$$
\prod_{\alpha=1}^{m} \frac{1}{\left|B_{j}^{k}\right|} \int_{B_{j}^{k}}\left|f_{\alpha}(y)\right| d y>2^{k} \quad j \geq 1 .
$$

We use a selection procedure as in [15, p. 372]. For convenience we set $b_{k}=\emptyset$ if $|k|>N$ and

$$
\Omega_{k}=\left\{\begin{array}{lll}
\bigcup_{s \geq k} \bigcup_{j} B_{j}^{s} & \text { when } & |k| \leq N \\
\emptyset & \text { when } & |k|>N .
\end{array}\right.
$$

Observe that these sets are decreasing in $k$, i.e., $\Omega_{k+1} \subset \Omega_{k}$.

We now distribute the sets in $\bigcup_{k} b_{k}$ over $\mu$ sequences $\left\{A_{i}(l)\right\}_{i \geq 1}, 0 \leq l \leq \mu-1$, where $\mu$ will be chosen momentarily to be an appropriately large natural number. Set $i_{0}(0)=1$. In the first $i_{1}(0)-i_{0}(0)$ entries of $\left\{A_{i}(0)\right\}_{i \geq 1}$, i.e., for

$$
i_{0}(0) \leq i<i_{1}(0),
$$

we place the elements of the sequence $b_{N}=\left\{B_{j}^{N}\right\}_{j \geq 1}$ in the order indicated by the index $j$. For the next $i_{2}(0)-i_{1}(0)$ entries of $\left\{A_{i}(0)\right\}_{i \geq 1}$, i.e., for

$$
i_{1}(0) \leq i<i_{2}(0)
$$

we place the elements of the sequence $b_{N-\mu}$. We continue in this way until we reach the first integer $m_{0}$ such that $N-m_{0} \mu \geq-N$, when we stop. For indices $i$ satisfying

$$
i_{m_{0}}(0) \leq i<i_{m_{0}+1}(0),
$$

we place in the sequence $\left\{A_{i}(0)\right\}_{i \geq 1}$ the elements of $b_{N-m_{0} \mu}$. The sequences $\left\{A_{i}(l)\right\}_{i \geq 1}$, $1 \leq l \leq \mu-1$, are defined similarly, starting from $b_{N-l}$ and using the families $b_{N-l-s \mu}$, $s=0,1, \cdots, m_{l}$, where $m_{l}$ is chosen so that $N-l-m_{l} \mu \geq-N$.

Since $\nu_{\vec{w}} \in A_{\infty, \mathcal{R}}, \nu_{\vec{w}}$ satisfies condition (A) by the remark made after Definition 5.2 and we can apply Lemma 5.1 to each $\left\{A_{i}(l)\right\}_{i \geq 1}$ for some fixed $0<\lambda<1$. Then we obtain sequences

$$
\left\{\tilde{A}_{i}(l)\right\}_{i \geq 1} \subset\left\{A_{i}(l)\right\}_{i \geq 1}, \quad 0 \leq l \leq \mu-1,
$$

which are $\lambda$-scattered with respect to the Lebesgue measure. In view of the definition of the set $\Omega_{k}$ and the construction of the families $\left\{A_{i}(l)\right\}_{i \geq 1}$, we can use Assertion 3) of Lemma 5.1 to obtain

$$
\nu_{\vec{w}}\left(\Omega_{k}\right) \leq c\left[\nu_{\vec{w}}\left(\Omega_{k+\mu}\right)+\nu_{\vec{w}}\left(\bigcup_{i_{m_{l}}(l) \leq i<i_{m_{l}+1}(l)} \tilde{A}_{i}(l)\right)\right] \leq c \nu_{\vec{w}}\left(\Omega_{k+\mu}\right)+c \sum_{i=i_{m_{l}}(l)}^{i_{m_{l}+1}(l)-1} \nu_{\vec{w}}\left(\tilde{A}_{i}(l)\right)
$$

if $k=N-l-m_{l} \mu$. It will be enough to consider these indices $k$ because the sets $\Omega_{k}$ are decreasing. 
Now, all the sets $\left\{\tilde{A}_{i}(l)\right\}_{i=i_{m}(l)}^{i_{m+1}(l)-1}$ belong to $b_{k}$ with $k=N-l-m_{l} \mu$ and therefore

$$
\prod_{\alpha=1}^{m} \frac{1}{\left|\tilde{A}_{i}(l)\right|} \int_{\tilde{A}_{i}(l)}\left|f_{\alpha}(x)\right| d x>2^{k}
$$

It now readily follows that

$$
\int_{2^{-N}<\mathcal{M}_{\mathcal{R}}(\vec{f}) \leq 2^{N+1}} \mathcal{M}_{\mathcal{R}}(\vec{f})(x)^{p} \nu_{\vec{w}}(x) d x \leq 2^{p} \sum_{k} 2^{k p} \nu_{\vec{w}}\left(\Omega_{k}\right)
$$

and then

$$
\begin{aligned}
& \sum_{k} 2^{k p} \nu_{\vec{w}}\left(\Omega_{k}\right) \leq c \sum_{k} 2^{k p} \nu_{\vec{w}}\left(\Omega_{k+\mu}\right)+c \sum_{l=0}^{\mu-1} \sum_{i \in I(l)} \nu_{\vec{w}}\left(\tilde{A}_{i}(l)\right)\left[\prod_{\alpha=1}^{m} \frac{1}{\left|\tilde{A}_{i}(l)\right|} \int_{\tilde{A}_{i}(l)}\left|f_{\alpha}\right| d x\right]^{p} \\
= & c 2^{-p \mu} \sum_{k} 2^{k p} \nu_{\vec{w}}\left(\Omega_{k}\right)+c \sum_{l=0}^{\mu-1} \sum_{i \in I(l)} \nu_{\vec{w}}\left(\tilde{A}_{i}(l)\right)\left(\prod_{\alpha=1}^{m} \frac{1}{\left|\tilde{A}_{i}(l)\right|} \int_{\tilde{A}_{i}(l)}\left|f_{\alpha}(x)\right| d x\right)^{p} .
\end{aligned}
$$

If we choose $\mu$ so large that $c 2^{-\mu p} \leq \frac{1}{2}$ and since everything involved is finite the first term on the right hand side can be subtracted from the left hand side. This yields

$$
\int_{2^{-N}<\mathcal{M}_{\mathcal{R}}(\vec{f}) \leq 2^{N+1}} \mathcal{M}_{\mathcal{R}}(\vec{f})^{p} \nu_{\vec{w}} d x \leq 2^{p+1} c \sum_{l=0}^{\mu-1} \sum_{i \in I(l)} \nu_{\vec{w}}\left(\tilde{A}_{i}(l)\right)\left(\prod_{\alpha=1}^{m} \frac{1}{\left|\tilde{A}_{i}(l)\right|} \int_{\tilde{A}_{i}(l)}\left|f_{\alpha}\right| d x\right)^{p} .
$$

We now proceed as in the proof of Theorem 2.3: for each $\alpha$ we use Hölder's inequality with exponents $p_{\alpha}^{\prime} r$ and $\left(p_{\alpha}^{\prime} r\right)^{\prime}$ to bound the previous expression on the right by

$$
\begin{aligned}
& 2^{p+1} c \sum_{l=0}^{\mu-1} \sum_{i \in I(l)}\left(\prod_{\alpha=1}^{m} \frac{1}{\left|\tilde{A}_{i}(l)\right|} \int_{\tilde{A}_{i}(l)}\left|f_{\alpha}\right| d x\right)^{p} \nu_{\vec{w}}\left(\tilde{A}_{i}(l)\right) \\
& \leq 2^{p+1} c \sum_{l=0}^{\mu-1} \sum_{i \in I(l)} \prod_{\alpha=1}^{m}\left(\frac{1}{\left|\tilde{A}_{i}(l)\right|} \int_{\tilde{A}_{i}(l)}\left|f_{\alpha}\right|^{\left(p_{\alpha}^{\prime} r\right)^{\prime}} w_{\alpha}^{\frac{\left(p_{\alpha}^{\prime} r\right)^{\prime}}{p_{\alpha}}} d x\right)^{\frac{p}{\left(p_{\alpha}^{\prime} r\right)^{\prime}}} \\
& \quad \times\left(\frac{1}{\left|\tilde{A}_{i}(l)\right|} \int_{\tilde{A}_{i}(l)} w_{\alpha}^{\frac{-p_{\alpha}^{\prime} r}{p_{\alpha}}} d x\right)^{\frac{p}{p_{\alpha}^{\prime} r}} \nu_{\vec{w}}\left(\tilde{A}_{i}(l)\right) .
\end{aligned}
$$

Recall that each $\sigma_{\alpha}=w_{\alpha}^{1-p_{\alpha}^{\prime}}$ satisfies the $A_{\infty, \mathcal{R}}$ condition and hence by the reverse Hölder inequality property of the basis $\mathcal{R}$ (see [8, p. 458]) there are constants $r_{\alpha}, c_{\alpha}>1$ such that

$$
\left(\frac{1}{|R|} \int_{R} \sigma_{\alpha}^{r_{\alpha}} d x\right)^{1 / r_{\alpha}} \leq c_{\alpha} \frac{1}{|R|} \int_{R} \sigma_{\alpha} d x \quad R \in \mathcal{R}
$$

Since $\vec{w} \in A_{\vec{P}, \mathcal{R}}$ we can therefore bound (5.6) by

$$
C \sum_{l=0}^{\mu-1} \sum_{i \in I(l)} \prod_{\alpha=1}^{m}\left(\frac{1}{\left|\tilde{A}_{i}(l)\right|} \int_{\tilde{A}_{i}(l)}\left|f_{\alpha}\right|^{\left(p_{\alpha}^{\prime} r\right)^{\prime}} w_{\alpha}^{\frac{\left(p_{\alpha}^{\prime} r\right)^{\prime}}{p_{\alpha}}} d x\right)^{\frac{p}{\left(p_{\alpha}^{\prime} r\right)^{\prime}}}\left|\tilde{A}_{i}(l)\right| .
$$


For each $l$ we let,

$$
E_{1}(l)=\tilde{A}_{1}(l) \quad \& \quad E_{i}(l)=\tilde{A}_{i}(l) \backslash \bigcup_{s<i} \tilde{A}_{s}(l) \quad i>1 .
$$

and we recall that the sequences $a(l)=\left\{\tilde{A}_{i}(l)\right\}_{i \in I(l)}$ are $\lambda$-scattered with respect to the Lebesgue measure, hence

$$
\left|\tilde{A}_{i}(l)\right| \leq \frac{1}{1-\lambda}\left|E_{i}(l)\right| \quad i>1
$$

Then we have the following estimate for (5.7)

$$
\frac{C}{1-\lambda} \sum_{l=0}^{\mu-1} \sum_{i \in I(l)} \prod_{\alpha=1}^{m}\left(\frac{1}{\left|\tilde{A}_{i}(l)\right|} \int_{\tilde{A}_{i}(l)}\left|f_{\alpha}\right|^{\left(p_{\alpha}^{\prime} r\right)^{\prime}} w_{\alpha}^{\frac{\left(p_{\alpha}^{\prime} r\right)^{\prime}}{p_{\alpha}}} d x\right)^{\frac{p}{\left(p_{\alpha}^{\prime} r\right)^{\prime}}}\left|E_{i}(l)\right| .
$$

Now, since the family $\left\{E_{i}(l)\right\}_{i, l}$ consists of pairwise disjoint sets and since

$$
\sum_{\alpha=1}^{m} \frac{p}{p_{\alpha}}=1
$$

using Hölder's inequality, we estimate (5.8) by a constant multiple of

$$
\begin{aligned}
& \sum_{l=0}^{\mu-1} \sum_{i \in I(l)} \int_{E_{i}(l)} \prod_{\alpha=1}^{m}\left(M_{\mathcal{R}}\left(\left|f_{\alpha}\right|^{\left(p_{\alpha}^{\prime} r\right)^{\prime}} w_{\alpha}^{\frac{\left(p_{\alpha}^{\prime} r\right)^{\prime}}{p_{\alpha}}}\right)\right)^{\frac{p}{\left(p_{\alpha}^{\prime} r\right)^{\prime}}} d x \\
\leq & c_{\mu} \int_{\mathbf{R}^{n}} \prod_{\alpha=1}^{m}\left(M_{\mathcal{R}}\left(\left|f_{\alpha}\right|^{\left(p_{\alpha}^{\prime} r\right)^{\prime}} w_{\alpha}^{\frac{\left(p_{\alpha}^{\prime} r\right)^{\prime}}{p_{\alpha}}}\right)\right)^{\frac{p}{\left(p_{\alpha}^{\prime} r\right)^{\prime}}} d x \\
\lesssim & \prod_{\alpha=1}^{m}\left(\int_{\mathbf{R}^{n}}\left(M_{\mathcal{R}}\left(\left|f_{\alpha}\right|^{\left(p_{\alpha}^{\prime} r\right)^{\prime}} w_{\alpha}^{\frac{\left(p_{\alpha}^{\prime} r\right)^{\prime}}{p_{\alpha}}}\right)\right)^{\frac{p_{\alpha}}{\left(p_{\alpha}^{\prime} r\right)^{\prime}}} d x\right)^{\frac{p}{p_{\alpha}}} \\
\lesssim & \prod_{\alpha=1}^{m}\left(\int_{\mathbf{R}^{n}}\left|f_{\alpha}\right|^{p_{\alpha}} w_{\alpha} d x\right)^{\frac{p}{p_{\alpha}}},
\end{aligned}
$$

since $p_{\alpha} /\left(p_{\alpha}^{\prime} r\right)^{\prime}>1$, which gives the desired strong-type estimate for (5.3). Letting $N \rightarrow \infty$ yields the claimed assertion of the theorem.

\section{Proof of the unweighted endpoint estimate for $\mathcal{M}_{\mathcal{R}}$}

In this section we prove Theorem 3.1. We begin by setting some notation and by proving several important ingredients required in the proof. 
6.1. Orlicz spaces and normalized measures. We need some basic facts from the theory of Orlicz spaces that we state without proof. We refer to the book of Rao and Ren [31] for the proofs and more information on Orlicz spaces. For a lively exposition of these spaces the reader may also consult the recent book by Wilson [34].

A Young function is a continuous, convex, increasing function $\Phi:[0, \infty) \rightarrow[0, \infty)$ with $\Phi(0)=0$ and such that $\Phi(t) \rightarrow \infty$ as $t \rightarrow \infty$. The properties of $\Phi$ easily imply that for $0<\epsilon<1$ and $t \geq 0$

$$
\Phi(\epsilon t) \leq \epsilon \Phi(t)
$$

The $\Phi$-norm of a function $f$ over a set $E$ with finite measure is defined by

$$
\|f\|_{\Phi, E}=\inf \left\{\lambda>0: \frac{1}{|E|} \int_{E} \Phi\left(\frac{|f(x)|}{\lambda}\right) d x \leq 1\right\} .
$$

We will use the fact that

$$
\|f\|_{\Phi, E}>1 \text { if and only if } \frac{1}{|E|} \int_{E} \Phi(|f(x)|) d x>1 .
$$

Associated with each Young function $\Phi$, one can define a complementary function

$$
\bar{\Phi}(s)=\sup _{t>0}\{s t-\Phi(t)\}
$$

for $s \geq 0$. Such $\bar{\Phi}$ is also a Young function and has the property that

$$
s t \leq C[\Phi(t)+\bar{\Phi}(s)]
$$

for all $s, t \geq 0$. Also the $\bar{\Phi}$-norms are related to the $L_{\Phi}$-norms via the the generalized Hölder inequality, namely

$$
\frac{1}{|E|} \int_{E}|f(x) g(x)| d x \leq 2\|f\|_{\Phi, E}\|g\|_{\bar{\Phi}, E}
$$

In this article we will be particularly interested in the pair of Young functions

$$
\Phi_{n}(t):=t[\log (e+t)]^{n-1} \quad \text { and } \quad \bar{\Phi}_{n}(t) \approx \Psi_{n}(t):=\exp \left(t^{\frac{1}{n-1}}\right)-1, \quad t \geq 0 .
$$

It is the case that the pair $\Phi_{n}, \Psi_{n}$ satisfies (6.5), see the article by Bagby [1], page 887 . Observe that the above function $\Phi_{n}$ is submultiplicative, a fact that will be used often in this article. That is, for $s, t>0$

$$
\Phi_{n}(s t) \leq c \Phi_{n}(s) \Phi_{n}(t)
$$

In Section 3 we introduced the function $\Phi^{(m)}:=\overbrace{\Phi \circ \Phi \circ \cdots \circ \Phi}^{m \text { times }}$ which is increasing with respect to $m \in \mathbb{N}$. 
6.2. Some Lemmas. We begin by proving some useful general lemmas about averaging functions.

Lemma 6.1. Let $\Phi$ be any Young function, then for any $f \geq 0$ and any measurable set $E$

$$
1<\|f\|_{\Phi, E} \quad \Rightarrow \quad\|f\|_{\Phi, E} \leq \frac{1}{|E|} \int_{E} \Phi(f(x)) d x .
$$

Proof. Indeed, by homogeneity this is equivalent to

$$
\left\|\frac{f}{\lambda_{f, E}}\right\|_{\Phi, E} \leq 1
$$

where

which is the same as

$$
\lambda_{f, E}=\frac{1}{|E|} \int_{E} \Phi(f(x)) d x
$$

$$
\frac{1}{|E|} \int_{E} \Phi\left(\frac{f(x)}{\lambda_{f, E}}\right) d x \leq 1
$$

by definition of the norm (6.2). In view of Property (6.1), it would be enough to show that

$$
\lambda_{f, E}=\frac{1}{|E|} \int_{E} \Phi(f(x)) d x \geq 1 .
$$

But this is the case by definition of the norm (Property (6.3))

$$
\|f\|_{\Phi, E}>1 \Longleftrightarrow \frac{1}{|E|} \int_{E} \Phi(f(x)) d x>1 .
$$

The following lemma is key for the main result. It should be mentioned that a different proof of this lemma will appear in a paper by Pérez, Pradolini, Torres and Trujillo-González [29]; see the proof of Theorem 4.1 therein.

Lemma 6.2. Let $\Phi$ be a submultiplicative Young function, let $m \in \mathbb{N}$ and let $E$ be any set. Then there is a constant $c$ such that whenever

$$
1<\prod_{i=1}^{m}\left\|f_{i}\right\|_{\Phi, E}
$$

holds, then

$$
\prod_{i=1}^{m}\left\|f_{i}\right\|_{\Phi, E} \leq c \prod_{i=1}^{m} \frac{1}{|E|} \int_{E} \Phi^{(m)}\left(f_{i}(x)\right) d x .
$$

Proof. a) The case $m=1$. This is the content of Lemma 6.1.

b) The case $m=2$. Fix functions for which (6.7) holds:

$$
1<\prod_{i=1}^{2}\left\|f_{i}\right\|_{\Phi, E}
$$


Without loss of generality we may assume that

$$
\left\|f_{1}\right\|_{\Phi, E} \leq\left\|f_{2}\right\|_{\Phi, E} .
$$

Observe that by (6.7) we must have $\left\|f_{2}\right\|_{\Phi, E}>1$.

Suppose first that $1 \leq\left\|f_{1}\right\|_{\Phi, E}$, then (6.8) follows from Lemma 6.1:

$$
1<\prod_{i=1}^{2}\left\|f_{i}\right\|_{\Phi, E} \leq \prod_{i=1}^{2} \frac{1}{|E|} \int_{E} \Phi\left(f_{i}(x)\right) d x
$$

with $m=1$ and $c=1$.

Assume now

$$
\left\|f_{1}\right\|_{\Phi, E} \leq 1 \leq\left\|f_{2}\right\|_{\Phi, E} .
$$

Then we have by Lemma 6.1, submultiplicativity and Jensen's inequality

$$
\begin{aligned}
1 & <\prod_{i=1}^{2}\left\|f_{i}\right\|_{\Phi, E} \\
& =\left\|f_{1}\right\|_{\Phi, E}\left\|f_{2}\right\|_{\Phi, E} \\
& =\left\|f_{1}\right\| f_{2}\left\|_{\Phi, E}\right\|_{\Phi, E} \\
& \leq c \frac{1}{|E|} \int_{E} \Phi\left(f_{1}(x)\left\|f_{2}\right\|_{\Phi, E}\right) d x \\
& \leq c \frac{1}{|E|} \int_{E} \Phi\left(f_{1}(x)\right) d x \Phi\left(\left\|f_{2}\right\|_{\Phi, E}\right) \\
& \leq c \frac{1}{|E|} \int_{E} \Phi\left(f_{1}(x)\right) d x \Phi\left(c \frac{1}{|E|} \int_{E} \Phi\left(f_{2}(x)\right) d x\right) \\
& \leq c \frac{1}{|E|} \int_{E} \Phi\left(f_{1}(x)\right) d x \frac{1}{|E|} \int_{E} \Phi^{(2)}\left(f_{2}(x)\right) d x \\
& \leq c \prod_{i=1}^{2} \frac{1}{|E|} \int_{E} \Phi^{(2)}\left(f_{i}(x)\right) d x
\end{aligned}
$$

which is exactly (6.8).

c) The case $m \geq 3$. By induction, assuming that the result holds for the integer $m-1 \geq 2$, we will prove it for $m$. Fix functions for which (6.7) holds:

$$
1<\prod_{i=1}^{m}\left\|f_{i}\right\|_{\Phi, E}
$$

and without loss of generality assume that

$$
\left\|f_{1}\right\|_{\Phi, E} \leq\left\|f_{2}\right\|_{\Phi, E} \leq \cdots \leq\left\|f_{m}\right\|_{\Phi, E} .
$$

Observe that we must have $\left\|f_{m}\right\|_{\Phi, E}>1$. 
If we suppose that $1 \leq\left\|f_{1}\right\|_{\Phi, E}$, then (6.8) follows directly from Lemma 6.1:

$$
1<\prod_{i=1}^{m}\left\|f_{i}\right\|_{\Phi, E} \leq \prod_{i=1}^{m} \frac{1}{|E|} \int_{E} \Phi\left(f_{i}(x)\right) d x
$$

with $c=1$ and $\Phi$ instead of $\Phi^{(2)}$.

Assume now that for some integer $k \in\{1,2, \ldots, m-1\}$ we have

$$
\left\|f_{1}\right\|_{\Phi, E} \leq\left\|f_{2}\right\|_{\Phi, E} \leq \cdots \leq\left\|f_{k}\right\|_{\Phi, E} \leq 1 \leq\left\|f_{k+1}\right\|_{\Phi, E} \leq \cdots \leq\left\|f_{m}\right\|_{\Phi, E} .
$$

Since

$$
1<\prod_{i=1}^{m}\left\|f_{i}\right\|_{\Phi, E}=\left\|f_{1}\right\|_{\Phi, E} \prod_{i=2}^{m}\left\|f_{i}\right\|_{\Phi, E},
$$

we must have $\prod_{i=2}^{m}\left\|f_{i}\right\|_{\Phi, E}>1$. Using the induction hypothesis we have

$$
\left\|f_{1}\right\|_{\Phi, E} \prod_{i=2}^{m}\left\|f_{i}\right\|_{\Phi, E} \leq c\left\|f_{1}\right\|_{\Phi, E} \prod_{i=2}^{m} \frac{1}{|E|} \int_{E} \Phi^{(m-1)}\left(f_{i}(x)\right) d x=\left\|f_{1} R\right\|_{\Phi, E},
$$

where $R=\prod_{i=2}^{m} \frac{1}{|E|} \int_{E} \Phi^{(m-1)}\left(f_{i}(x)\right) d x$. Applying Lemma 6.1 to the function $f_{1} R$ we obtain by submultiplicativity and Jensen's inequality

$$
\begin{aligned}
\left\|f_{1} R\right\|_{\Phi, E} & \leq c \frac{1}{|E|} \int_{E} \Phi\left(f_{1}(x) R\right) d x \\
& \leq c \frac{1}{|E|} \int_{E} \Phi\left(f_{1}(x)\right) d x \Phi(R) \\
& \leq c \frac{1}{|E|} \int_{E} \Phi\left(f_{1}(x)\right) d x \prod_{i=2}^{m} \Phi\left(\frac{1}{|E|} \int_{E} \Phi^{(m-1)}\left(f_{i}(x)\right) d x\right) \\
& \leq c \frac{1}{|E|} \int_{E} \Phi\left(f_{1}(x)\right) d x \prod_{i=2}^{m} \frac{1}{|E|} \int_{E} \Phi^{(m)}\left(f_{i}(x)\right) d x .
\end{aligned}
$$

Combining this result with (6.9) we deduce

$$
\prod_{i=1}^{m}\left\|f_{i}\right\|_{\Phi, E} \leq c \prod_{i=1}^{m} \frac{1}{|E|} \int_{E} \Phi^{(m)}\left(f_{i}(x)\right) d x
$$

thus proving (6.8).

\subsection{The proof of the endpoint estimates.}

Proof of Theorem 3.1. By homogeneity, positivity of the operator, and the doubling property of $\Phi_{n}$, it is enough to prove

$$
\left|\left\{x \in \mathbf{R}^{n}: \mathcal{M}_{\mathcal{R}}(\vec{f})(x)>1\right\}\right| \leq C\left\{\prod_{j=1}^{m} \int_{\mathbf{R}^{n}} \Phi_{n}^{(m)}\left(f_{j}(x)\right) d x\right\}^{1 / m},
$$

for some constant $C$ independent of the vector of nonnegative functions $\vec{f}=\left(f_{1}, \cdots, f_{m}\right)$. 
Let $E=\left\{x \in \mathbf{R}^{n}: \mathcal{M}_{\mathcal{R}}(\vec{f})(x)>1\right\}$, then by the continuity property of the Lebesgue measure we can find a compact set $K$ such that $K \subset E$ and

$$
|K| \leq|E| \leq 2|K|
$$

Such a compact set $K$ can be covered with a finite collection of rectangles $\left\{R_{j}\right\}_{j=1}^{N}$ such that

$$
\prod_{i=1}^{m} \frac{1}{\left|R_{j}\right|} \int_{R_{j}} f_{i}(y) d y>1, \quad j=1, \cdots, N .
$$

We will use the following version of the Córdoba-Fefferman rectangle covering lemma [5] due to Bagby ([1] Theorem. $4.1(\mathrm{C}))$ : there are dimensional positive constants $\delta, c$ and a subfamily $\left\{\widetilde{R}_{j}\right\}_{j=1}^{\ell}$ of $\left\{R_{j}\right\}_{j=1}^{N}$ satisfying

$$
\left|\bigcup_{j=1}^{N} R_{j}\right| \leq c\left|\bigcup_{j=1}^{\ell} \widetilde{R}_{j}\right|
$$

and

$$
\int_{\bigcup_{j=1}^{\ell} \widetilde{R}_{j}} \exp \left(\delta \sum_{j=1}^{\ell} \chi_{\widetilde{R}_{j}}(x)\right)^{\frac{1}{n-1}} d x \leq 2\left|\bigcup_{j=1}^{\ell}\right| \widetilde{R}_{j} \mid
$$

Setting $\widetilde{E}=\bigcup_{j=1}^{\ell} \widetilde{R}_{j}$ and recalling that $\Psi_{n}(t)=\exp \left(t^{\frac{1}{n-1}}\right)-1$ the latter inequality is

$$
\frac{1}{|\widetilde{E}|} \int_{\widetilde{E}} \Psi_{n}\left(\delta \sum_{j=1}^{\ell} \chi_{\widetilde{R}_{j}}(x)\right) d x \leq 1
$$

which is equivalent to

$$
\left\|\sum_{j=1}^{\ell} \chi_{\widetilde{R}_{j}}\right\|_{\Psi_{n}, \widetilde{E}} \leq \frac{1}{\delta}
$$

by the definition of the norm. Now, since

$$
|E| \leq 2|K| \leq C|\widetilde{E}|
$$


we can use (6.11) and Hölder's inequality as follows

$$
\begin{aligned}
|\widetilde{E}| & =\left|\bigcup_{j=1}^{\ell} \widetilde{R}_{j}\right| \\
& \leq \sum_{j=1}^{\ell}\left|\widetilde{R}_{j}\right| \\
& \leq \sum_{j=1}^{\ell}\left(\prod_{i=1}^{m} \int_{\widetilde{R}_{j}} f_{i}(y) d y\right)^{\frac{1}{m}} \\
& \leq\left(\prod_{i=1}^{m} \sum_{j=1}^{\ell} \int_{\widetilde{R}_{j}} f_{i}(y) d y\right)^{\frac{1}{m}} \\
& \leq\left(\prod_{i=1}^{m} \int_{\bigcup_{j=1}^{\ell} \widetilde{R}_{j}} \sum_{j=1}^{\ell} \chi_{\widetilde{R}_{j}}(y) f_{i}(y) d y\right)^{\frac{1}{m}} \\
& =\left(\prod_{i=1}^{m} \int_{\widetilde{E}_{j=1}}^{\ell} \sum_{\widetilde{R}_{j}}(y) f_{i}(y) d y\right)^{\frac{1}{m}} .
\end{aligned}
$$

By this inequality and (6.6), we deduce

$$
\begin{aligned}
1 & \leq \prod_{i=1}^{m} \frac{1}{|\widetilde{E}|} \int_{\widetilde{E}} \sum_{j=1}^{\ell} \chi_{\widetilde{R}_{j}}(y) f_{i}(y) d y \\
& \leq \prod_{i=1}^{m}\left\|\sum_{j=1}^{\ell} \chi_{\widetilde{R}_{j}}\right\|_{\Psi_{n}, \widetilde{E}}\left\|f_{i}\right\|_{\Phi_{n}, \widetilde{E}} \\
& \leq \prod_{i=1}^{m} \frac{1}{\delta}\left\|f_{i}\right\|_{\Phi_{n}, \widetilde{E}} \\
& =\prod_{i=1}^{m}\left\|\frac{f_{i}}{\delta}\right\|_{\Phi_{n}, \widetilde{E}} .
\end{aligned}
$$

Finally, it is enough to apply Lemma 6.2 and that $\Phi_{n}^{(m)}$ is submultiplicative to conclude the proof of (6.10), which is the main part of the theorem.

We now turn to the claimed sharpness of the theorem. In the case $m=n=2$, we need to show that the estimate

$$
\left|\left\{x \in \mathbf{R}^{2}: \mathcal{M}_{\mathcal{R}}(f, g)(x)>\alpha^{2}\right\}\right| \leq C\left\{\int_{\mathbf{R}^{2}} \Phi_{2}\left(\frac{|f(x)|}{\alpha}\right) d x\right\}^{\frac{1}{2}}\left\{\int_{\mathbf{R}^{2}} \Phi_{2}\left(\frac{|g(x)|}{\alpha}\right) d x\right\}^{\frac{1}{2}}
$$

cannot hold for $\alpha>0$ and functions $f, g$ with a constant $C$ independent of these parameters. 
For $N=1,2, \ldots$, consider the functions

$$
f=\chi_{[0,1]^{2}} \quad \text { and } \quad g_{N}=N \chi_{[0,1]^{2}}
$$

and the parameter $\alpha=\frac{1}{10}$. Then the left hand side of the inequality reduces to

$$
\begin{aligned}
\left|\left\{x \in \mathbb{R}^{2}: \mathcal{M}_{\mathcal{R}}\left(f, g_{N}\right)(x)>\frac{1}{100}\right\}\right| & =\left|\left\{x \in \mathbb{R}^{2}: M_{\mathcal{R}}\left(\chi_{[0,1]^{2}}\right)(x)>\frac{1}{10 \sqrt{N}}\right\}\right| \\
& \approx \sqrt{N}(\log N),
\end{aligned}
$$

where the last estimate is a simple calculation concerning the strong maximal function (i.e., the case $m=1$ ) that can be found, for instance, in [9, p. 384, Exercise 10.3.1].

However, the right hand side is equal to

$$
C\left(\Phi_{2}(1 / \alpha)\right)^{1 / 2}\left(\Phi_{2}(N / \alpha)\right)^{1 / 2}=C\left(\Phi_{2}(10)\right)^{1 / 2}\left(\Phi_{2}(10 N)\right)^{1 / 2} \approx \sqrt{N \log N}
$$

and obviously it cannot control the left hand side for $N$ large.

For general $m$, the vector $\vec{f}$ with

$$
f_{1}=f_{2}=\cdots=f_{m-1}=\chi_{[0,1]^{2}} \quad \text { and } \quad f_{m}=N \chi_{[0,1]^{2}}
$$

also provides a counterexample.

\section{INTERPOLATION BETWEEN DISTRIBUTIONAL ESTIMATES}

We have seen that bi(sub)linear operators satisfy certain distributional estimates that are variations of the usual weak $L^{p}$ estimates. Multilinear interpolation between a set of restricted weak type conditions is a well understood topic, but the issue of multilinear interpolation between more general distributional estimates has not been studied. We begin this section with the following interpolation result between distributional estimates. The result is not bilinear per se, as the first function remains in the same space during the interpolation, however, it only requires two initial conditions instead of three required in the classical real-method bilinear interpolation, see for instance [10]. Also the next result can be applied to the maximal function $\mathcal{M}_{\mathcal{R}}$, yielding an $L^{1} \times L^{p}$ estimate for it.

Proposition 7.1. Let $T$ be a bisublinear operator. Suppose that there exists $B_{1}>0$ such that for all $f, g \in L_{\Phi_{n}}^{1}\left(\mathbf{R}^{n}\right)$ and all $\alpha>0$ we have

$$
\left|\left\{x \in \mathbf{R}^{n}: T(f, g)(x)>\alpha\right\}\right| \leq \sqrt{B_{1}\left\|\Phi_{n}\left(\frac{|f|}{\sqrt{\alpha}}\right)\right\|_{L^{1}\left(\mathbf{R}^{n}\right)}\left\|\Phi_{n}\left(\frac{|g|}{\sqrt{\alpha}}\right)\right\|_{L^{1}\left(\mathbf{R}^{n}\right)}}
$$

Also suppose that there exists $B_{2}>0$ such that for all $f \in L_{\Phi_{n}}^{1}\left(\mathbf{R}^{n}\right), g \in L^{\infty}\left(\mathbf{R}^{n}\right)$ and all $\alpha>0$,

$$
\left|\left\{x \in \mathbf{R}^{n}: T(f, g)(x)>\alpha\right\}\right| \leq B_{2}\left\|\Phi_{n}\left(\frac{|f|}{\sqrt{\alpha}}\right)\right\|_{L^{1}\left(\mathbf{R}^{n}\right)} \Phi_{n}\left(\frac{\|g\|_{L^{\infty}\left(\mathbf{R}^{n}\right)}}{\sqrt{\alpha}}\right) .
$$


Then, for all $f \in L_{\Phi_{n}}^{1}\left(\mathbf{R}^{n}\right), g \in L_{\Phi_{n}}^{p}\left(\mathbf{R}^{n}\right)$ with $p \in(1, \infty)$, and all $\alpha>0$,

$$
\begin{aligned}
\mid\left\{x \in \mathbf{R}^{n}:\right. & T(f, g)(x)>\alpha\} \mid \\
\leq & C\left\{B_{1}^{\frac{1}{p}} B_{2}^{\frac{p-1}{p}}\left\|\Phi_{n}\left(\frac{|f|}{\sqrt{\alpha}}\right)\right\|_{L^{1}\left(\mathbf{R}^{n}\right)}\left\|\Phi_{n}\left(\frac{|g|}{\sqrt{\alpha}}\right)\right\|_{L^{p}\left(\mathbf{R}^{n}\right)}\right\}^{\frac{p}{p+1}},
\end{aligned}
$$

where $C>0$ depends only on $n$.

Proof. For any $\alpha>0$, we split $g$ as $g=g_{\alpha}+g^{\alpha}$ where

$$
g_{\alpha}:=g \chi_{\{|g| \leq \epsilon \sqrt{\alpha / 2}\}}, \quad \text { and } \quad g^{\alpha}:=g \chi_{|g|>\epsilon \sqrt{\alpha / 2}}
$$

where $\epsilon$ is a positive quantity to be determined. Then,

$$
\begin{aligned}
\left|\left\{x \in \mathbf{R}^{n}: T(f, g)(x)>\alpha\right\}\right| \\
\quad \leq\left|\left\{x \in \mathbf{R}^{n}: T\left(f, g^{\alpha}\right)(x)>\alpha / 2\right\}\right|+\left|\left\{x \in \mathbf{R}^{n}: T\left(f, g_{\alpha}\right)(x)>\alpha / 2\right\}\right| \\
\quad:=L_{1}+L_{2} .
\end{aligned}
$$

Since $\Phi_{n}$ is a strictly increasing function on $(0, \infty)$ and $p>1$, we have

$$
\begin{aligned}
\left\|\Phi_{n}\left(\frac{\left|g^{\alpha}\right|}{\sqrt{\alpha / 2}}\right)\right\|_{L^{1}\left(\mathbf{R}^{n}\right)} & =\int_{\{|g|>\epsilon \sqrt{\alpha / 2}\}} \Phi_{n}\left(\frac{|g(x)|}{\sqrt{\alpha / 2}}\right) d x \\
& \leq \frac{1}{\Phi_{n}(\epsilon)^{p-1}} \int_{\{|g|>\epsilon \sqrt{\alpha / 2}\}} \Phi_{n}\left(\frac{|g(x)|}{\sqrt{\alpha / 2}}\right)^{p} d x \\
& \leq C \frac{\left\|\Phi_{n}\left(\frac{|g|}{\sqrt{\alpha}}\right)\right\|_{L^{p}\left(\mathbf{R}^{n}\right)}^{p}}{\Phi_{n}(\epsilon)^{p-1}}
\end{aligned}
$$

and hence, by (7.1),

$$
\begin{aligned}
L_{1} & \leq \sqrt{B_{1}\left\|\Phi_{n}\left(\frac{|f|}{\sqrt{\alpha / 2}}\right)\right\|\left\|_{L^{1}\left(\mathbf{R}^{n}\right)}\right\| \Phi_{n}\left(\frac{\left|g^{\alpha}\right|}{\sqrt{\alpha / 2}}\right) \|_{L^{1}\left(\mathbf{R}^{n}\right)}} \\
& \leq C \sqrt{\frac{B_{1}}{\Phi_{n}(\epsilon)^{p-1}}\left\|\Phi_{n}\left(\frac{|f|}{\sqrt{\alpha}}\right)\right\|_{L^{1}\left(\mathbf{R}^{n}\right)}\left\|\Phi_{n}\left(\frac{|g|}{\sqrt{\alpha}}\right)\right\|_{L^{p}\left(\mathbf{R}^{n}\right)}^{p}} .
\end{aligned}
$$

Also, by (7.2),

$$
L_{2} \leq\left\|\Phi_{n}\left(\frac{|f|}{\sqrt{\alpha / 2}}\right)\right\|_{L^{1}\left(\mathbf{R}^{n}\right)} \Phi_{n}\left(\frac{\left\|g_{\alpha}\right\|_{L^{\infty}\left(\mathbf{R}^{n}\right)}}{\sqrt{\alpha / 2}}\right) \leq C B_{2} \Phi_{n}(\epsilon)\left\|\Phi_{n}\left(\frac{|f|}{\sqrt{\alpha}}\right)\right\|_{L^{1}\left(\mathbf{R}^{n}\right)} .
$$


Again, using $\Phi_{n}$ is a strictly increasing function on $(0, \infty)$ with $\Phi_{n}(0)=0$ and $\Phi_{n}(\infty)=$ $\infty$, we can choose $\epsilon \in(0, \infty)$ such that

$$
\Phi_{n}(\epsilon)=\left(\frac{B_{1}\left\|\Phi_{n}\left(\frac{|g|}{\sqrt{\alpha}}\right)\right\|_{L^{p}\left(\mathbf{R}^{n}\right)}^{p}}{B_{2}^{2}\left\|\Phi_{n}\left(\frac{|f|}{\sqrt{\alpha}}\right)\right\|_{L^{1}\left(\mathbf{R}^{n}\right)}}\right)^{\frac{1}{p+1}} .
$$

For such an $\epsilon$, both $L_{1}$ and $L_{2}$ are bounded by

$$
C B_{1}^{\frac{1}{p+1}} B_{2}^{1-\frac{2}{p+1}}\left(\left\|\Phi_{n}\left(\frac{|f|}{\sqrt{\alpha}}\right)\right\|_{L^{1}\left(\mathbf{R}^{n}\right)}\left\|\Phi_{n}\left(\frac{|g|}{\sqrt{\alpha}}\right)\right\|_{L^{p}\left(\mathbf{R}^{n}\right)}\right)^{\frac{p}{p+1}}
$$

which proves (7.3).

Corollary 7.2. Let $p \in(1, \infty)$. Then, there exists a positive constant $C$ depending only on the dimension $n$ such that for all $f \in L_{\Phi_{n}^{(2)}}^{1}\left(\mathbf{R}^{n}\right), g \in L_{\Phi_{n}^{(2)}}^{p}\left(\mathbf{R}^{n}\right)$ and all $\alpha>0$,

$$
\begin{aligned}
\mid\{x & \left.\in \mathbf{R}^{n}: \mathcal{M}_{\mathcal{R}}(f, g)(x)>\alpha\right\} \mid \\
& \leq C\left\{\int_{\mathbf{R}^{n}} \Phi_{n}^{(2)}\left(\frac{|f(x)|}{\sqrt{\alpha}}\right) d x\right\}^{\frac{p}{p+1}}\left\{\int_{\mathbf{R}^{n}} \Phi_{n}^{(2)}\left(\frac{|g(x)|}{\sqrt{\alpha}}\right)^{p} d x\right\}^{\frac{1}{p+1}} .
\end{aligned}
$$

Our last result yields strong type bounds for a bilinear operator with the initial assumption of two weak type estimates and a third distributional estimate. This result provides a generalization of the well-known real (or Marcinkiewicz) bilinear interpolation theorem.

Theorem 7.3. Let $1<s_{1}<s_{2}<\infty$ and $1 / s_{1}+1 / s_{2}=1 / s$. Suppose that a bisublinear operator $T$ maps $L^{s_{1}} \times L^{s_{2}} \rightarrow L^{s, \infty}$ with norm $B_{1}$, it maps $L^{s_{2}} \times L^{s_{1}} \rightarrow L^{s, \infty}$ with norm $B_{2}$, it maps $L^{2 s} \times L^{2 s}$ to $L^{s, \infty}$ with norm $B$, and it also satisfies the following distributional estimate at the endpoint $(1,1,1 / 2)$

$$
\left|\left\{\left|T\left(f_{1}, f_{2}\right)\right|>\lambda\right\}\right| \leq A\left(\int \Phi\left(\frac{f_{1}}{\sqrt{\lambda}}\right) d x\right)^{\frac{1}{2}}\left(\int \Phi\left(\frac{f_{2}}{\sqrt{\lambda}}\right) d x\right)^{\frac{1}{2}},
$$

where $\Phi$ is a nonnegative function that satisfies $\Phi(0)=0$, and

$$
\int_{0}^{1} \lambda^{\alpha} \Phi\left(\frac{1}{\lambda}\right) d \lambda<\infty
$$

for all $\alpha>0$. Then, $T: L^{p_{1}} \times L^{p_{2}} \rightarrow L^{p}$ for all indices $p_{1}, p_{2}, p$ with $1 / p_{1}+1 / p_{2}=$ $1 / p$ and $\left(1 / p_{1}, 1 / p_{2}, 1 / p\right)$ is in the open convex hull of the points $\left(1 / s_{1}, 1 / s_{2}, 1 / s\right)$, $\left(1 / s_{2}, 1 / s_{1}, 1 / s\right)$ and $\left(1 / s_{1}, 1 / s_{1}, 2 / s_{1}\right)$.

Proof. We begin by observing that if $T$ is actually linear in every entry (instead of sublinear), then the condition that $T$ maps $L^{2 s} \times L^{2 s}$ to $L^{s, \infty}$ is redundant as it can be deduced from the hypotheses that $T$ maps $L^{s_{1}} \times L^{s_{2}} \rightarrow L^{s, \infty}$ and that it maps $L^{s_{2}} \times L^{s_{1}} \rightarrow L^{s, \infty}$ via bilinear complex interpolation since the point $(1 / 2 s, 1 / 2 s, 1 / s)$ lies halfway between $\left(1 / s_{1}, 1 / s_{2}, 1 / s\right)$, and $\left(1 / s_{2}, 1 / s_{1}, 1 / s\right)$. 
We also note that in the desired range of exponents in the conclusion of the theorem we always have $s_{1} / 2<p<s<s_{2} / 2$. We will show the result holds when $p_{1}=p_{2}=2 p$ and $(p, p, p / 2)$ is in the claimed open convex hull. Boundedness for the remaining triples follows then by real bilinear interpolation; see [10].

We fix two functions $f_{1}, f_{2}$ in $L^{2 p}$ with norm equal to 1 and write

$$
f_{1, \lambda}=f_{1} \chi_{\left|f_{1}\right|>\sqrt{\lambda}}, \quad f_{1}^{\lambda}=f_{1} \chi_{\left|f_{1}\right| \leq \sqrt{\lambda}}
$$

and likewise for $f_{2}$.

We now estimate the measure

$$
\left|\left\{\left|T\left(f_{1}, f_{2}\right)\right|>4 \lambda\right\}\right| \leq I(\lambda)+I I(\lambda)+I I I(\lambda)+I V(\lambda),
$$

where

$$
\begin{aligned}
I(\lambda) & =\left|\left\{\left|T\left(f_{1, \lambda}, f_{2, \lambda}\right)\right|>\lambda\right\}\right|, \\
I I(\lambda) & =\left|\left\{\left|T\left(f_{1, \lambda}, f_{2}^{\lambda}\right)\right|>\lambda\right\}\right|, \\
I I I(\lambda) & =\left|\left\{\left|T\left(f_{1}^{\lambda}, f_{2, \lambda}\right)\right|>\lambda\right\}\right|, \\
I V(\lambda) & =\left|\left\{\left|T\left(f_{1}^{\lambda}, f_{2}^{\lambda}\right)\right|>\lambda\right\}\right| .
\end{aligned}
$$

First we take a look at

$$
\int_{0}^{\infty} \lambda^{p-1} I(\lambda) d \lambda
$$

which is bounded by

$$
\begin{aligned}
& \int_{0}^{\infty} \lambda^{p-1} A\left(\int \Phi\left(\frac{f_{1, \lambda}}{\sqrt{\lambda}}\right) d x\right)^{\frac{1}{2}}\left(\int \Phi\left(\frac{f_{2, \lambda}}{\sqrt{\lambda}}\right) d x\right)^{\frac{1}{2}} d \lambda \\
& \leq A \prod_{i=1}^{2}\left(\int_{0}^{\infty} \lambda^{p-1} \int_{\left|f_{i}\right|>\sqrt{\lambda}} \Phi\left(\frac{f_{i}}{\sqrt{\lambda}}\right) d x d \lambda\right)^{\frac{1}{2}} \\
& =A \prod_{i=1}^{2}\left(\iint_{\lambda=0}^{\left|f_{i}(x)\right|^{2}} \lambda^{p-1} \Phi\left(\frac{f_{i}}{\sqrt{\lambda}}\right) d \lambda d x\right)^{\frac{1}{2}} \\
& =2 A \prod_{i=1}^{2}\left(\int\left|f_{i}(x)\right|^{2 p} \int_{\lambda=0}^{1} \lambda^{2 p-1} \Phi\left(\frac{1}{\lambda}\right) d \lambda d x\right)^{\frac{1}{2}} \\
& =C_{p} A \prod_{i=1}^{2}\left\|f_{i}\right\|_{L^{2 p}}^{p}=C_{p} A,
\end{aligned}
$$

where we have made some simple changes of variables and the convergence of the integral is due to the fact that $p>1 / 2$. 
We now split $p-s=s\left(\frac{p}{s_{1}}-\frac{1}{2}\right)+s\left(\frac{p}{s_{2}}-\frac{1}{2}\right)$ and we look at

which can be estimated by

$$
\int_{0}^{\infty} \lambda^{p-1} I I(\lambda) d \lambda
$$

$$
\begin{aligned}
\int_{0}^{\infty} \lambda^{p-s} B_{1}^{s}\left(\int\left|f_{1, \lambda}\right|^{s_{1}} d x\right)^{\frac{s}{s_{1}}}\left(\int\left|f_{2}^{\lambda}\right|^{s_{2}} d x\right)^{\frac{s}{s_{2}}} \frac{d \lambda}{\lambda} \\
\leq B_{1}^{s}\left(\int_{0}^{\infty} \lambda^{s\left(\frac{p}{s_{1}}-\frac{1}{2}\right) \frac{s_{1}}{s}} \int_{\left|f_{1}\right|>\sqrt{\lambda}}\left|f_{1}\right|^{s_{1}} d x \frac{d \lambda}{\lambda}\right)^{\frac{s}{s_{1}}} \\
\times\left(\int_{0}^{\infty} \lambda^{s\left(\frac{p}{s_{2}}-\frac{1}{2}\right) \frac{s_{2}}{s}} \int_{\left|f_{2}\right| \leq \sqrt{\lambda}}\left|f_{2}\right|^{s_{2}} d x \frac{d \lambda}{\lambda}\right)^{\frac{s}{s_{2}}} \\
\leq B_{1}^{s}\left(\int\left|f_{1}(x)\right|^{s_{1}} \int_{0}^{\left|f_{1}(x)\right|^{2}} \lambda^{s_{1}\left(\frac{p}{s_{1}}-\frac{1}{2}\right)} \frac{d \lambda}{\lambda} d x\right)^{\frac{s}{s_{1}}} \\
\quad \times\left(\int\left|f_{2}(x)\right|^{s_{2}} \int_{\left|f_{2}(x)\right|^{2}}^{\infty} \lambda^{s_{2}\left(\frac{p}{s_{2}}-\frac{1}{2}\right)} \frac{d \lambda}{\lambda} d x\right)^{\frac{s}{s_{2}}} \\
\leq B_{1}^{s} C\left(s_{1}, s_{2}, p\right)\left\|f_{1}\right\|_{L^{2 p}}^{\frac{2 p s}{s_{1}}}\left\|f_{2}\right\|_{L^{2 p}}^{\frac{2 p s}{s_{2}}} \\
=B_{1}^{s} C\left(s_{1}, s_{2}, p\right)
\end{aligned}
$$

and both integrals converge since $s_{1}<2 p<s_{2}$. The term involving $I I I(\lambda)$ is treated similarly using the bound $B_{2}$.

Finally we look at

$$
\int_{0}^{\infty} \lambda^{p-1} I V(\lambda) d \lambda
$$

which is bounded by

$$
\begin{aligned}
& \int_{0}^{\infty} \lambda^{p-1-s} B^{s}\left(\int\left|f_{1}^{\lambda}\right|^{2 s} d x\right)^{\frac{s}{2 s}}\left(\int\left|f_{2}^{\lambda}\right|^{2 s} d x\right)^{\frac{s}{2 s}} d \lambda \\
& \leq B^{s} \prod_{i=1}^{2}\left(\int_{0}^{\infty} \lambda^{p-1-s} \int_{\left|f_{i}\right| \leq \sqrt{\lambda}}\left|f_{i}(x)\right|^{2 s} d x d \lambda\right)^{\frac{1}{2}} \\
& =B^{s} \prod_{i=1}^{2}\left(\int\left|f_{i}(x)\right|^{2 s} \int_{\left|f_{i}(x)\right|^{2}}^{\infty} \lambda^{p-s-1} d \lambda d x\right)^{\frac{1}{2}} \\
& =B^{s} \prod_{i=1}^{2}\left(\int\left|f_{i}(x)\right|^{2 s}\left|f_{i}(x)\right|^{2 p-2 s} \int_{\lambda=1}^{\infty} \lambda^{p-s-1} d \lambda d x\right)^{\frac{1}{2}} \\
& =B^{s} C(p, s) \prod_{i=1}^{2}\left\|f_{i}\right\|_{L^{2 p}}^{p}=B^{s} C(p, s),
\end{aligned}
$$

where the convergence of the integral is due to the fact that $p<s$. 
Remark 7.4. It is not hard to see that a similar result can be obtained in the above theorem if instead of the symmetric weak type estimates $L^{s_{1}} \times L^{s_{2}} \rightarrow L^{s, \infty}$ and $L^{s_{2}} \times$ $L^{s_{1}} \rightarrow L^{s, \infty}$ one has $L^{t_{1}} \times L^{t_{2}} \rightarrow L^{s, \infty}$ and $L^{r_{1}} \times L^{r_{2}} \rightarrow L^{s, \infty}$ where $1 / t_{1}+1 / t_{2}=$ $1 / r_{1}+1 / r_{2}=1 / s, t_{1} / 2<s<t_{2} / 2$ and $r_{1} / 2>s>r_{2} / 2$. For example, if $r_{1} \geq t_{2}$, one gets strong bounds on the open triangle with vertices $\left(1 / t_{1}, 1 / t_{2}, 1 / s\right),\left(1 / r_{1}, 1 / r_{2}, 1 / s\right)$ and $\left(1 / t_{1}, 1 / t_{1}, 2 / t_{1}\right)$.

Corollary 7.5. Suppose a bisublinear operator $T$ maps $L^{s_{1}} \times L^{s_{2}} \rightarrow L^{s, \infty}$ for all $1<s_{1}, s_{2}, s<\infty$ with $1 / s_{1}+1 / s_{2}=1 / s$ and also satisfies the endpoint distributional estimate of Theorem 7.3. Then $T: L^{p_{1}} \times L^{p_{2}} \rightarrow L^{p}$ for all $1 / p_{1}+1 / p_{2}=1 / p$ with $1<p_{1}, p_{2}<\infty$ and $1 / 2<p<\infty$.

The situation in Corollary 7.5 arises in the study of the bilinear strong maximal function but also in the the study of certain commutators of bilinear singular integrals and pointwise multiplication with functions in $B M O$. For a bilinear Calderón-Zygmund operator $T$ as in [11] and $b_{1}, b_{2}$ in $B M O$ consider

$$
T_{b_{1}, b_{2}}\left(f_{1}, f_{2}\right)=b_{1} T\left(f_{1}, f_{2}\right)-T\left(b_{1} f_{1}, f_{2}\right)+b_{2} T\left(f_{1}, f_{2}\right)-T\left(f_{1}, b_{2} f_{2}\right) .
$$

It was shown in [30] that $T_{b_{1}, b_{2}}: L^{s_{1}} \times L^{s_{2}} \rightarrow L^{s}$ for all $1<s_{1}, s_{2}, s<\infty$ with $1 / s_{1}+1 / s_{2}=1 / s$. The proof in [30] was based on weighted estimates and cannot be extended to $1 / 2<s \leq 1$. A question was asked then what kind of endpoint estimate the operator $T_{b_{1}, b_{2}}$ may satisfy. Later on in [18] a distributional estimate as in Theorem 7.3 was obtained with

$$
\Phi(t)=t\left(1+\log ^{+}(t)\right),
$$

but the question still remained open about how to interpolate using such an estimate. A different method was used in [18] to obtain the result $T_{b_{1}, b_{2}}: L^{s_{1}} \times L^{s_{2}} \rightarrow L^{s}$ for $1 / 2<s \leq 1$, but we now see from Corollary 7.5 that the result can also be obtained by interpolation. This puts in evidence that the distributional endpoint estimates achieved in this article are the appropriate ones from the point of view of interpolation.

It is also interesting to point out that the distributional estimates we use are not quite $L \log L$-type norms. In fact, even in the linear case and estimate $L \log L \rightarrow L^{1, \infty}$ together with a strong type $(p, p)$ for $p>1$ do not produce in general $(q, q)$ estimates for $1<q<p$, unless the operator in question is a translation invariant one in a compact setting. See $[33]^{1}$

\section{REFERENCES}

[1] R. Bagby, Maximal functions and rearrangements: some new proofs, Indiana Univ. Math. J. 32 (1983), 879-891.

[2] R. R. Coifman and Y. Meyer, Commutateurs d'intégrales singulières et opérateurs multilinéaires, Ann. Inst. Fourier (Grenoble) 28 (1978), 177-202.

[3] R. R. Coifman and Y. Meyer, Au délà des opérateurs pseudo-différentiels, Astérisque No. 57, Societé Mathématique de France, 1979.

\footnotetext{
${ }^{1}$ We would like to thank M. J. Carro, M. Cwikel, and N. Kalton for pointing out this reference and facts and for interesting conversations on the subject.
} 
[4] A. Córdoba, On the Vitali covering properties of a differentiation basis, Studia Math. 57 (1976), 91-95.

[5] A. Córboda and R. Fefferman, A geometric proof of the strong maximal theorem, Ann. Math. 102 (1975), 95-100.

[6] D. Cruz-Uribe, J. M. Martell and C. Pérez, Sharp two-weight inequalities for singular integrals, with applications to the Hilbert transform and the Sarason conjecture, Adv. in Math. 216 (2007), 647-676.

[7] R. Fefferman, Multiparameter Fourier Analysis, Annals of Math. Studies 112, Beijing Lectures in Harmonic Analysis, pp. 47-130, Princeton University Press, Princeton 1986.

[8] J. García-Cuerva and J. L. Rubio de Francia, Weighted Norm Inequalities and Related Topics, North Holland, Amsterdam, 1985.

[9] L. Grafakos, Modern Fourier Analysis, Second Edition, Springer-Verlag, Graduate Texts in Mathematics, No. 250, Springer, New York, 2009.

[10] L. Grafakos and N. Kalton, Some remarks on multilinear maps and interpolation, Math. Ann. 319 (2001), 151-180.

[11] L. Grafakos and R. H. Torres, Multilinear Calderón-Zygmund theory, Adv. in Math. 165 (2002), $124-164$.

[12] L. Grafakos and R. H. Torres, Maximal operator and weighted norm inequalities for multilinear singular integrals, Indiana Univ. Math. J. 51 (2002), 1261-1276.

[13] P. Hagelstein and A. Stokolos, An extension of the Córdoba-Fefferman theorem on the equivalence between the boundedness of certain classes of maximal and multiplier operators, C. R. Acad. Sci. Paris, Ser. I 346 (2008), 1063-1065.

[14] P. Hagelstein and A. Stokolos, Tauberian conditions for Geometric Maximal operators, Trans. Amer. Math. Soc. 361 (2009), 3031-3040.

[15] B. Jawerth, Weighted inequalities for maximal operators: linearization, localization, and factorization, Amer. J. Math. 108 (1986), 361-414.

[16] B. Jawerth and A. Torchinsky, The strong maximal function with respect to measures, Studia Math. 80 (1984), 261-285.

[17] B. Jessen, J. Marcinkiewicz and A. Zygmund, Note on the differentiability of multiple integrals, Fund. Math. 25 (1935), 217-234.

[18] A. K. Lerner, S. Ombrosi, C. Pérez, R. H. Torres and R. Trujillo-González, New maximal functions and multiple weights for the multilinear Calderón-Zygmund theory, Adv. in Math. 220 (2009), 1222-1264.

[19] A. K. Lerner, An elementary approach to several results on the Hardy-Littlewood maximal operator, Proc. Amer. Math. Soc. 136 (2008), 2829-2833.

[20] K. Moen, Weighted inequalities for multilinear fractional integral operators, Collect. Math. 60 (2009), 213-238.

[21] B. Muckenhoupt, Weighted norm inequalities for the Hardy maximal function, Trans. Amer. Math. Soc. 165 (1972), 207-226.

[22] C. J. Neugebauer, Inserting $A_{p}$-weights, Proc. Amer. Math. Soc. 87 (1983), 644-648.

[23] C. Pérez, Weighted norm inequalities for general maximal operators, Publ. Mat. 35 (1991), 169186.

[24] C. Pérez, A remark on weighted inequalities for general maximal operators, Proc. Amer. Math. Soc. 119 (1993), 1121-1126.

[25] C. Pérez, On sufficient conditions for the boundedness of the Hardy-Littlewood maximal operator between weighted $L^{p}$-spaces with different weights, Proc. London Math. Soc. (3) 71 (1995), 135-157.

[26] C. Pérez, Weighted norm inequalities for singular integral operators, J. London Math. Soc. 49 (1994), 296-308. 
[27] C. Pérez, Two weighted inequalities for potential and fractional type maximal operators, Indiana Univ. Math. J. 43 (1994), 663-683.

[28] C. Pérez, Endpoint estmates for commutators of singular integral operators, J. Funct. Anal. 128 (1995), 163-185.

[29] C. Pérez, G. Pradolini, R. H. Torres and R. Trujillo-González, End-point estimates for iterated commutators of multilinear singular integrals, arXiv:1004.4976.

[30] C. Pérez and R. H. Torres, Sharp maximal function estimates for multilinear singular integrals, Contemp. Math. 320 (2003), 323-331.

[31] M. M. Rao and Z. D. Ren, Theory of Orlicz Spaces, Marcel Dekker, New York, 1991.

[32] E. T. Sawyer, A characterization of a two weight norm weight inequality for maximal operators, Studia Math. 75 (1982), 1-11.

[33] T. Tao, A converse extrapolation theorem for translation-invariant operators, J. Funct. Anal. 180 (2001), 1-10.

[34] M. Wilson, Littlewood-Paley Theory and Exponential-Square Integrability, Lectures Notes in Math. 1924, Springer-Verlag, Berlin 2008.

Loukas Grafakos, Department of Mathematics, University of Missouri, Columbia, MO 65211, USA

E-mail address: grafakosl@missouri.edu

Liguang Liu, Department of Mathematics, School of Information, Renmin UniverSity of China, BeiJing 100872, China

E-mail address: liguangbnu@gmail.com

Carlos Pérez, Departamento De Análisis Matemático, Facultad de Matemáticas, Universidad De Sevilla, 41080 Sevilla, Spain.

E-mail address: carlosperez@us.es

Rodolfo H. Torres, Department of Mathematics, University of Kansas, 405 Snow Hall 1460 JayhaWK Blvd, LaWrence, Kansas 66045-7523, USA.

E-mail address: torres@math.ku.edu 